\title{
Labelings for Decreasing Diagrams
}

\author{
Harald Zankl • Bertram Felgenhauer • Aart Middeldorp
}

Received: 29 November 2013 / Accepted: 8 October 2014 / Published online: 17 December 2014

(C) The Author(s) 2014. This article is published with open access at Springerlink.com

\begin{abstract}
This article is concerned with automating the decreasing diagrams technique of van Oostrom for establishing confluence of term rewrite systems. We study abstract criteria that allow to lexicographically combine labelings to show local diagrams decreasing. This approach has two immediate benefits. First, it allows to use labelings for linear rewrite systems also for left-linear ones, provided some mild conditions are satisfied. Second, it admits an incremental method for proving confluence which subsumes recent developments in automating decreasing diagrams. The techniques proposed in the article have been implemented and experimental results demonstrate how, e.g., the rule labeling benefits from our contributions.
\end{abstract}

Keywords Term rewriting $\cdot$ Confluence $\cdot$ Decreasing diagrams $\cdot$ Automation

\section{Introduction}

Confluence is an important property of rewrite systems since it ensures unique normal forms. It is decidable in the presence of termination [14] and implied by orthogonality [21] or restricted joinability conditions on the critical pairs [12, 15, 19, 20, 24]. Recently, there is a renewed interest in confluence research, with a strong emphasis on automation. As one application we mention [22], where automated confluence tools are employed for proving soundness of abstract forms of reduction in solving the typing problem.

This research is supported by FWF (Austrian Science Fund) project P22467.

H. Zankl $(\bowtie) \cdot$ B. Felgenhauer · A. Middeldorp

Institute of Computer Science, University of Innsbruck, 6020 Innsbruck, Austria

e-mail: harald.zankl@uibk.ac.at

B. Felgenhauer

e-mail: bertram.felgenhauer@uibk.ac.at

A. Middeldorp

e-mail: aart.middeldorp@uibk.ac.at 
The decreasing diagrams technique of van Oostrom [16] is a complete method for showing confluence of countable abstract rewrite systems. The main idea of the approach is to show confluence by establishing local confluence under the side condition that rewrite steps of the joining sequences must decrease with respect to some well-founded order. For term rewrite systems however, the main problem for automation of decreasing diagrams is that in general infinitely many local peaks must be considered. To reduce this problem to a finite set of local peaks one can label rewrite steps with functions that satisfy special properties. In [17] van Oostrom presented the rule labeling that allows to conclude confluence of linear rewrite systems by checking decreasingness of the critical peaks (those emerging from critical overlaps). The rule labeling has been implemented by Aoto [1] and Hirokawa and Middeldorp [11]. Already in [17] van Oostrom presented constraints that allow to apply the rule labeling to left-linear systems. This approach has been implemented and extended by Aoto [1]. Our framework subsumes the above ideas.

The contributions of this article comprise the extraction of abstract constraints on a labeling such that for a (left-)linear rewrite system decreasingness of the (parallel) critical peaks ensures confluence. We show that the rule labeling adheres to our constraints and present additional labeling functions. Furthermore such labeling functions can be combined lexicographically to obtain new labeling functions satisfying our constraints. This approach allows the formulation of an abstract criterion that makes virtually every labeling function for linear rewrite systems also applicable to left-linear systems. Consequently, confluence of the TRS in Example 1 can be established automatically, e.g., by the rule labeling, while current approaches based on the decreasing diagrams technique $[1,11]$ as well as other confluence criteria like Knuth and Bendix' criterion or orthogonality (and its refinements) fail.

Example 1 Consider the TRS $\mathcal{R}$ (Cops \#60) ${ }^{1}$ consisting of the rules

\begin{tabular}{|c|c|c|}
\hline $1: x+(y+z$ & $\rightarrow(x+y)+z$ & $6: x \times y$ \\
\hline$(x+y)+$ & $\rightarrow x+(y+z)$ & 7: $\mathrm{s}(x)+y \rightarrow x+\mathrm{s}(y)$ \\
\hline $\mathrm{sq}(x)$ & $\rightarrow x \times x$ & $8: x+\mathrm{s}(y) \rightarrow \mathrm{s}(x)+y$ \\
\hline $\mathrm{sq}(\mathrm{s}(x))$ & $\rightarrow(x \times x)+\mathrm{s}(x+x)$ & $9: x \times \mathrm{s}(y) \rightarrow x+(x \times y)$ \\
\hline$x+y$ & $\rightarrow y+x$ & 10: $\mathrm{s}(x) \times y \rightarrow(x \times y)+y$ \\
\hline
\end{tabular}

This system is locally confluent since all its 34 critical pairs are joinable.

The remainder of this article is organized as follows. After recalling preliminaries in Section 2 we present constraints (on a labeling) such that decreasingness of the critical peaks ensures confluence for (left-)linear rewrite systems in Section 3. Three of these constraints are based on relative termination while the fourth employs persistence. We focus on parallel rewriting in Section 4. The merits of these approaches are assessed in Section 5 by discussing the relationship to the recent literature. Implementation issues are addressed in Section 6 before Section 7 gives an empirical evaluation of our results. Section 8 concludes.

This article is an updated and extended version of [31], which presents the first incremental approach for labeling decreasing diagrams. Besides a number of small improvements, the article contains three new major contributions:

- Section 3.2.3, presenting a new labeling measuring the contracted redex,

${ }^{1}$ COnfluence ProblemS, see http://coco.nue.riec.tohoku.ac.jp/problems/. 
- Section 3.2.4, which uses persistence to enhance the applicability of L-labelings for left-linear systems,

- Section 4, which studies parallel rewriting to make any weak LL-labeling applicable to showing confluence of left-linear systems without additional (relative termination) constraints.

The latter generalizes and incorporates recent findings from [6], which studies the rule labeling for parallel rewriting.

\section{Preliminaries}

We assume familiarity with term rewriting [5, 23].

Let $\mathcal{F}$ be a signature and let $\mathcal{V}$ be a set of variables disjoint from $\mathcal{F}$. By $\mathcal{T}(\mathcal{F}, \mathcal{V})$ we denote the set of terms over $\mathcal{F}$ and $\mathcal{V}$. The expression $|t|_{x}$ indicates how often variable $x$ occurs in term $t$. Positions are strings of natural numbers, i.e., elements of $\mathbb{N}_{+}^{*}$. The set of positions of a term $t$ is defined as $\mathcal{P} \operatorname{os}(t)=\{\epsilon\}$ if $t$ is a variable and as $\mathcal{P} \operatorname{os}(t)=\{\epsilon\} \cup\{i q \mid$ $1 \leqslant i \leqslant n$ and $\left.q \in \mathcal{P o s}\left(t_{i}\right)\right\}$ if $t=f\left(t_{1}, \ldots, t_{n}\right)$. We write $p \leqslant q$ if $q=p p^{\prime}$ for some position $p^{\prime}$, in which case $q \backslash p$ is defined to be $p^{\prime}$. Furthermore $p<q$ if $p \leqslant q$ and $p \neq q$. Finally, $p \| q$ if neither $p \leqslant q$ nor $q<p$. Positions are used to address subterm occurrences. The subterm of $t$ at position $p \in \mathcal{P}$ os $(t)$ is defined as $\left.t\right|_{p}=t$ if $p=\epsilon$ and as $\left.t\right|_{p}=\left.t_{i}\right|_{q}$ if $p=i q$. We write $u \unlhd t$ if $u$ is a subterm of $t$ and $s[t]_{p}$ for the result of replacing $\left.s\right|_{p}$ with $t$ in $s$. The set of function symbol positions $\mathcal{P o s}_{\mathcal{F}}(t)$ is $\left\{p \in \mathcal{P}\right.$ os $\left.(t)|t|_{p} \notin \mathcal{V}\right\}$ and $\operatorname{Pos}_{\mathcal{V}}(t)=\mathcal{P}$ os $(t) \backslash \mathcal{P o s}_{\mathcal{F}}(t)$. The set of variables occurring in a term $t$ is denoted by $\mathcal{V} \operatorname{ar}(t)$. We let $\left.t\right|_{P}=\left\{\left.t\right|_{p} \mid p \in P\right\}$ if $t$ is a term and $P$ a set of positions.

A rewrite rule is a pair of terms $(l, r)$, written $l \rightarrow r$, such that $l$ is not a variable and all variables in $r$ are contained in $l$. A rewrite rule $l \rightarrow r$ is duplicating if $|l|_{x}<|r|_{x}$ for some $x \in \mathcal{V}$. A term rewrite system (TRS) is a signature together with a finite set of rewrite rules over this signature. In the sequel signatures are implicit. By $\mathcal{R}_{d}$ and $\mathcal{R}_{n d}$ we denote the duplicating and non-duplicating rules of a TRS $\mathcal{R}$, respectively. A rewrite relation is a binary relation on terms that is closed under contexts and substitutions. For a TRS $\mathcal{R}$ we define $\rightarrow_{\mathcal{R}}$ to be the smallest rewrite relation that contains $\mathcal{R}$. As usual $\rightarrow^{=}, \rightarrow^{+}$, and $\rightarrow^{*}$ denotes the reflexive, transitive, and reflexive and transitive closure of $\rightarrow$, respectively.

A relative TRS $\mathcal{R} / \mathcal{S}$ is a pair of TRSs $\mathcal{R}$ and $\mathcal{S}$ with the induced rewrite relation $\rightarrow \mathcal{R} / \mathcal{S}=\rightarrow_{\mathcal{S}}^{*} \cdot \rightarrow_{\mathcal{R}} \cdot \rightarrow_{\mathcal{S}}^{*}$. Sometimes we identify a TRS $\mathcal{R}$ with the relative TRS $\mathcal{R} / \varnothing$ and vice versa. A TRS $\mathcal{R}$ (relative TRS $\mathcal{R} / \mathcal{S})$ is terminating if $\rightarrow_{\mathcal{R}}\left(\rightarrow_{\mathcal{R} / \mathcal{S}}\right)$ is wellfounded. Two relations $\geqslant$ and $>$ are called compatible if $\geqslant \cdot>\cdot \geqslant \subseteq>$. A monotone reduction pair $(\geqslant,>)$ consists of a preorder $\geqslant$ and a well-founded order $>$ such that $\geqslant$ and $>$ are compatible and closed under contexts and substitutions. A reduction pair $(\geqslant,>)$ is called simple if $f\left(s_{1}, \ldots, s_{n}\right) \geqslant s_{i}$ for all $1 \leqslant i \leqslant n$. We recall how to prove relative termination incrementally according to Geser [8].

Theorem 2 A relative TRS $\mathcal{R} / \mathcal{S}$ is terminating if $\mathcal{R}=\varnothing$ or there exists a monotone reduction pair $(\geqslant,>)$ such that $\mathcal{R} \cup \mathcal{S} \subseteq \geqslant$ and $(\mathcal{R} \backslash>) /(\mathcal{S} \backslash>)$ is terminating.

A critical overlap $\left(l_{1} \rightarrow r_{1}, p, l_{2} \rightarrow r_{2}\right)_{\mu}$ of a TRS $\mathcal{R}$ consists of variants $l_{1} \rightarrow r_{1}$ and $l_{2} \rightarrow r_{2}$ of rewrite rules of $\mathcal{R}$ without common variables, a position $p \in \mathcal{P o s}_{\mathcal{F}}\left(l_{2}\right)$, and a most general unifier $\mu$ of $l_{1}$ and $\left.l_{2}\right|_{p}$. If $p=\epsilon$ then we require that $l_{1} \rightarrow r_{1}$ and $l_{2} \rightarrow r_{2}$ 
are not variants. From a critical overlap $\left(l_{1} \rightarrow r_{1}, p, l_{2} \rightarrow r_{2}\right)_{\mu}$ we obtain a critical peak $l_{2} \mu\left[r_{1} \mu\right]_{p} \leftarrow l_{2} \mu \rightarrow r_{2} \mu$ and a critical pair $l_{2} \mu\left[r_{1} \mu\right]_{p} \leftarrow \rtimes \rightarrow r_{2} \mu$.

If $l \rightarrow r \in \mathcal{R}$ and $p$ is a position, we call the pair $\pi=\langle p, l \rightarrow r\rangle$ a redex pattern, and write $l_{\pi}, r_{\pi}, p_{\pi}$ for its left-hand side, right-hand side, and position, respectively. We write $\rightarrow^{\pi}$ (or $\rightarrow^{p_{\pi}, l_{\pi} \rightarrow r_{\pi}}$ ) for a rewrite step at position $p_{\pi}$ using the rule $l_{\pi} \rightarrow r_{\pi}$. A redex pattern $\pi$ matches a term $t$ if $\left.t\right|_{p_{\pi}}$ is an instance of $l_{\pi}$. If $\pi$ matches $t$, there is a unique reduct $t^{\pi}$ with $t \rightarrow^{\pi} t^{\pi}$.

Let $\pi_{1}$ and $\pi_{2}$ be redex patterns that match a common term. They are called parallel $\left(\pi_{1} \| \pi_{2}\right)$ if $p_{\pi_{1}} \| p_{\pi_{2}}$. If $p_{\pi_{2}} \leqslant p_{\pi_{1}}$ and $p_{\pi_{1}} \backslash p_{\pi_{2}} \in \mathcal{P}_{\mathrm{os}_{\mathcal{F}}}\left(l_{\pi_{2}}\right)$ then $\pi_{1}$ and $\pi_{2}$ overlap critically; otherwise they are called orthogonal $\left(\pi_{1} \perp \pi_{2}\right)$. Note that $\pi_{1} \| \pi_{2}$ implies $\pi_{1} \perp \pi_{2}$. We write $P \perp Q$ if $\pi \perp \pi^{\prime}$ for all $\pi \in P$ and $\pi^{\prime} \in Q$ and similarly $P \| Q$ if $\pi \| \pi^{\prime}$ for all $\pi \in P$ and $\pi^{\prime} \in Q$. If $P$ is a set of pairwise parallel redex patterns matching a term $t$, we denote by $t \rightarrow^{P} t^{\prime}$ the parallel rewrite step from $t$ to $t^{\prime}$ by $P$, where $t^{\prime}=t^{\pi_{1} \cdots \pi_{n}}$ if $P=\left\{\pi_{1}, \ldots, \pi_{n}\right\}$. We allow $P$ to be abbreviated to a set of positions in $t \rightarrow^{P} t^{\prime}$.

We write $\left\langle A,\left\{\rightarrow_{\alpha}\right\}_{\alpha \in I}\right\rangle$ to denote the $\operatorname{ARS}\langle A, \rightarrow\rangle$ where $\rightarrow$ is the union of $\rightarrow_{\alpha}$ for all $\alpha \in I$. Let $\left\langle A,\left\{\rightarrow_{\alpha}\right\}_{\alpha \in I}\right\rangle$ be an ARS and let $\geqslant$ and $>$ be relations on $I$. We write $\rightarrow{ } \alpha_{1} \cdots \alpha_{n}$ for the union of $\rightarrow_{\beta}$ where $\beta<\alpha_{i}$ for some $1 \leqslant i \leqslant n$. We call $\rightarrow_{\alpha}$ and $\rightarrow_{\beta}$ decreasing (with respect to $\geqslant$ and $>$ ) if

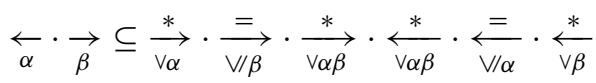

An ARS $\left\langle A,\left\{\rightarrow_{\alpha}\right\}_{\alpha \in I}\right\rangle$ is decreasing if there exists a preorder $\geqslant$ and a well-founded order $>$ such that $\geqslant$ and $>$ are compatible and $\rightarrow_{\alpha}$ and $\rightarrow \beta$ are decreasing for all $\alpha, \beta \in I$ with respect to $\geqslant$ and $>$.

The following theorem is a reformulation of a result obtained by van Oostrom [16] (where $\geqslant$ is the identity relation). While allowing a preorder $\geqslant$ does not add power, it is more convenient for our purposes.

Theorem 3 Every decreasing ARS is confluent.

\section{Labelings for Rewrite Steps}

In this section we present constraints (on a labeling) such that decreasingness of the critical peaks ensures confluence of linear (Section 3.1) and left-linear (Section 3.2) TRSs. Furthermore, we show that if two labelings satisfy these conditions then also their lexicographic combination satisfies them.

For a local peak

$$
t=s\left[r_{1} \sigma\right]_{p} \leftarrow s\left[l_{1} \sigma\right]_{p}=s=s\left[l_{2} \sigma\right]_{q} \rightarrow s\left[r_{2} \sigma\right]_{q}=u
$$

there are three possibilities (modulo symmetry):

(a) $\quad p \| q$ (parallel),

(b) $q \leqslant p$ and $p \backslash q \in \mathcal{P o s}_{\mathcal{F}}\left(l_{2}\right)$ (critical overlap),

(c) $q<p$ and $p \backslash q \notin \mathcal{P o s}_{\mathcal{F}}\left(l_{2}\right)$ (variable overlap).

These cases are visualized in Fig. 1. Figure 1a shows the shape of a local peak where the steps take place at parallel positions. Here we have $s \rightarrow^{p, l_{1} \rightarrow r_{1}} t$ and $u \rightarrow^{p, l_{1} \rightarrow r_{1}} v$ as well as $s \rightarrow^{q, l_{2} \rightarrow r_{2}} u$ and $t \rightarrow^{q, l_{2} \rightarrow r_{2}} v$, i.e., the steps drawn at opposing sides in the diagram are due to the same rules. The question mark in Fig. $1 \mathrm{~b}$ conveys that joinability of critical 


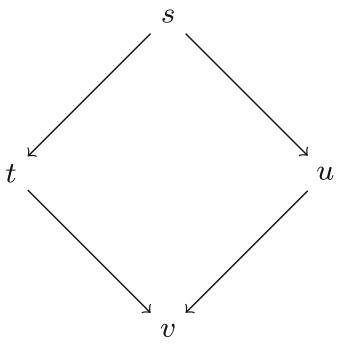

(a) (parallel)

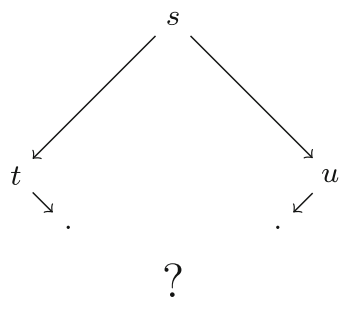

(b) (critical overlap)

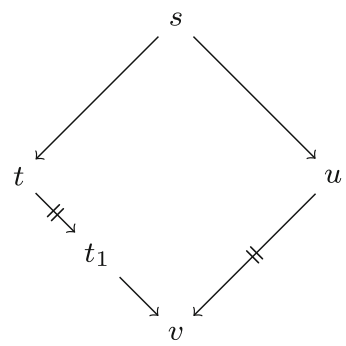

(c) (variable overlap)

Fig. 1 Three kinds of local peaks

overlaps may depend on auxiliary rules. Variable overlaps (Fig. 1c) can again be joined by the rules involved in the diverging step. More precisely, if $q^{\prime}$ is the unique position in $\operatorname{Pos}_{\mathcal{V}}\left(l_{2}\right)$ such that $q q^{\prime} \leqslant p, x=\left.l_{2}\right|_{q^{\prime}},\left|l_{2}\right|_{x}=m$, and $\left|r_{2}\right|_{x}=n$ then we have $t \rightarrow{ }_{l_{1} \rightarrow r_{1}}^{m-1} t_{1}$, $t_{1} \rightarrow_{l_{2} \rightarrow r_{2}} v$, and $u \rightarrow_{l_{1} \rightarrow r_{1}}^{n} v$.

Labelings are used to compare rewrite steps. In the sequel we denote the set of all rewrite steps for a TRS $\mathcal{R}$ by TRS $\mathcal{R}$ and elements from this set by capital Greek letters $\Gamma$ and $\Delta$. Furthermore if $\Gamma=s \rightarrow^{p, l \rightarrow r} t$ then $C[\Gamma \sigma]$ denotes the rewrite step $C[s \sigma] \rightarrow p^{\prime} p, l \rightarrow r C[t \sigma]$ for any substitution $\sigma$ and context $C$ with $\left.C\right|_{p^{\prime}}=\square$.

Definition 4 Let $\mathcal{R}$ be a TRS. A labeling function $\ell: \Lambda_{\mathcal{R}} \rightarrow W$ is a mapping from rewrite steps into some set $W$. A labeling $(\ell, \geqslant,>)$ for $\mathcal{R}$ consists of a labeling function $\ell$, a preorder $\geqslant$, and a well-founded order $>$ such that $\geqslant$ and $>$ are compatible and for all rewrite steps $\Gamma, \Delta \in \Lambda_{\mathcal{R}}$, contexts $C$ and substitutions $\sigma$ :

1. $\ell(\Gamma) \geqslant \ell(\Delta)$ implies $\ell(C[\Gamma \sigma]) \geqslant \ell(C[\Delta \sigma])$, and

2. $\ell(\Gamma)>\ell(\Delta)$ implies $\ell(C[\Gamma \sigma])>\ell(C[\Delta \sigma])$.

All labelings we present satisfy $>\subseteq \geqslant$, which allows to avoid tedious case distinctions, and we assume this property henceforth. We do so without loss of generality, because $\left((>\cup \geqslant)^{*},>\right)$ satisfies the conditions of Definition 4 if $(\geqslant,>)$ does.

In the sequel $W, \geqslant$, and $>$ are left implicit when clear from the context and a labeling is identified with the labeling function $\ell$. We use the terminology that a labeling $\ell$ is monotone and stable if properties 1 and 2 of Definition 4 hold. Abstract labels, i.e., labels that are unknown, are represented by lowercase Greek letters $\alpha, \beta, \gamma$, and $\delta$. We write $s \rightarrow_{\alpha}^{\pi} t$ (or simply $\left.s \rightarrow_{\alpha} t\right)$ if $\ell\left(s \rightarrow^{\pi} t\right)=\alpha$. Often we leave the labeling $\ell$ implicit and just attach labels to arrows. A local peak $t \leftarrow s \rightarrow u$ is called decreasing for $\ell$ if there are labels $\alpha$ and $\beta$ such that $t_{\alpha} \leftarrow s \rightarrow_{\beta} u$, and $\rightarrow_{\alpha}$ and $\rightarrow_{\beta}$ are decreasing with respect to $\geqslant$ and $>$. To employ Theorem 3 for TRSs, decreasingness of the $\operatorname{ARS}\left\langle\mathcal{T}(\mathcal{F}, \mathcal{V}),\left\{\rightarrow_{w}\right\}_{w \in W}\right\rangle$ must be shown.

In this article we investigate conditions on a labeling such that local peaks according to (parallel) and (variable overlap) are decreasing automatically. This is desirable since in general there are infinitely many local peaks corresponding to these cases (even if the underlying TRS has finitely many rules). There are also infinitely many local peaks according to (critical overlap) in general, but for a finite TRS they are captured by the finitely many critical overlaps. Still, it is undecidable if they are decreasingly joinable [11]. 
For later reference, Fig. 2 shows labeled local peaks for the case (parallel) (Fig. 2a) and (variable overlap) if the rule $l_{2} \rightarrow r_{2}$ in local peak (1) is linear (Fig. 2b) and left-linear (Fig. 2c), respectively. In Fig. 2c the expression $\bar{\gamma}$ denotes a sequence of labels $\gamma_{1}, \ldots, \gamma_{n}$. In the subsequent analysis we will always use the fact that the local peaks in Fig. 2 can be closed by the rules involved in the peak (applied at opposing sides in the diagram).

\subsection{Linear TRSs}

The next definition presents sufficient abstract conditions on a labeling such that local peaks according to the cases (parallel) and (variable-linear) in Fig. 2 are decreasing. We use the observation that for linear TRSs the (parallel) case can be seen as an instance of the (variable-linear) case to shorten proofs.

Definition 5 Let $\ell$ be a labeling for a TRS $\mathcal{R}$. We call $\ell$ an L-labeling (for $\mathcal{R}$ ) if for local peaks according to (parallel) and (variable-linear) we have $\alpha \geqslant \gamma$ and $\beta \geqslant \delta$ in Fig. 2a and Fig. 2b, respectively.

The local diagram in Fig. 3a visualizes the conditions on an L-labeling more succinctly. We will use L-labelings also for left-linear TRSs, where no conditions are required for local peaks different from (parallel) and (variable-linear). We call the critical peaks of a TRS $\mathcal{R} \Phi$-decreasing if there exists a $\Phi$-labeling $\ell$ for $\mathcal{R}$ such that the critical peaks of $\mathcal{R}$ are decreasing for $\ell$. In the sequel we will introduce further labelings, e.g., LL-labelings and weak LL-labelings. The placeholder $\Phi$ avoids the need for repeating the definition of decreasingness for these labelings.

The next theorem states that L-labelings may be used to show confluence of linear TRSs.

Theorem 6 Let $\mathcal{R}$ be a linear TRS. If the critical peaks of $\mathcal{R}$ are L-decreasing then $\mathcal{R}$ is confluent.

Proof By assumption the critical peaks of $\mathcal{R}$ are decreasing for some L-labeling $\ell$. We establish confluence of $\mathcal{R}$ by Theorem 3, i.e., show decreasingness of the ARS $\left\langle\mathcal{T}(\mathcal{F}, \mathcal{V}), \rightarrow_{\mathcal{R}}\right\rangle$ where rewrite steps are labeled according to $\ell$. Since $\mathcal{R}$ is linear, local peaks have the shape (parallel), (variable-linear), or (critical overlap). By definition of an L-labeling the former two are decreasing. Now consider a local peak according to (critical overlap), i.e., for the local peak (1) we have $q \leqslant p$ and $p \backslash q \in \mathcal{P o s}_{\mathcal{F}}\left(l_{2}\right)$.

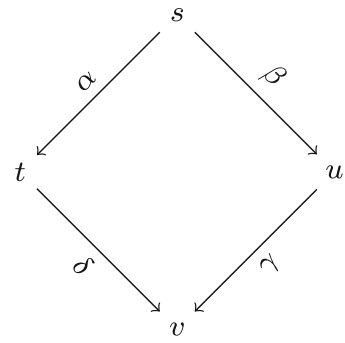

(a) (parallel)

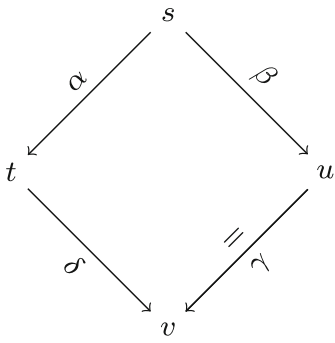

(b) (variable-linear)

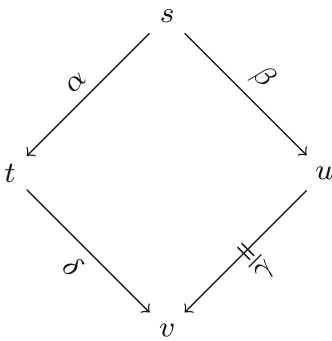

(c) (variable-left-linear)

Fig. 2 Labeled local peaks 


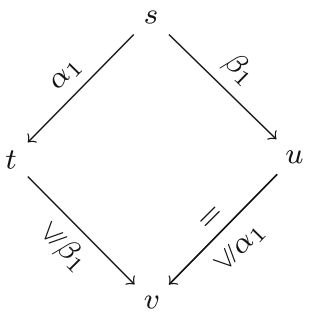

(a) Labeling $\ell_{1}$

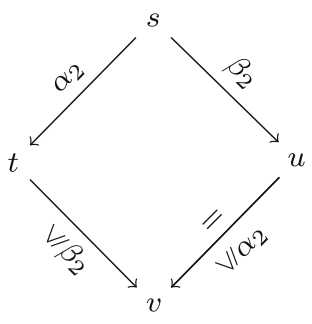

(b) Labeling $\ell_{2}$

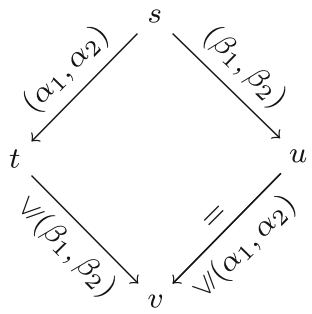

(c) Labeling $\ell_{1} \times \ell_{2}$

Fig. 3 Lexicographic combination of L-labelings

Let $p^{\prime}=p \backslash q$. Then $\left.\left.\left.t\right|_{q} \leftarrow s\right|_{q} \rightarrow u\right|_{q}$ must be an instance of a critical peak $l_{2} \mu\left[r_{1} \mu\right]_{p^{\prime}} \leftarrow$ $l_{2}\left[l_{1} \mu\right]_{p^{\prime}}=l_{2} \mu \rightarrow r_{2} \mu$ which is decreasing by assumption. By monotonicity and stability of $\ell$ we obtain decreasingness of the local peak (1).

We recall the rule labeling of van Oostrom [17], parametrized by a mapping $i: \mathcal{R} \rightarrow \mathbb{N}$. Often $i$ is left implicit. The rule labeling satisfies the constraints of an L-labeling.

Lemma 7 Let $\mathcal{R}$ be a TRS and $\ell_{r l}^{i}\left(s \rightarrow^{\pi} t\right)=i\left(l_{\pi} \rightarrow r_{\pi}\right)$. Then $\left(\ell_{r l}^{i}, \geqslant_{\mathbb{N}},>_{\mathbb{N}}\right)$ is an L-labeling for $\mathcal{R}$.

Proof First we show that $\left(\ell_{\mathrm{rl}}^{i}, \geqslant_{\mathbb{N}},>_{\mathbb{N}}\right)$ is a labeling. The preorder $\geqslant_{\mathbb{N}}$ and the well-founded order $>_{\mathbb{N}}$ are compatible. Furthermore $\ell_{\mathrm{rl}}^{i}\left(s \rightarrow^{\pi} t\right)=i\left(l_{\pi} \rightarrow r_{\pi}\right)$ which ensures monotonicity and stability of $\ell_{\mathrm{rl}}^{i}$. Hence $\left(\ell_{\mathrm{rl}}^{i}, \geqslant_{\mathbb{N}},>_{\mathbb{N}}\right)$ is a labeling. Next we show the properties demanded in Definition 5. For local peaks according to cases (parallel) and (variable-linear) we recall that the steps drawn at opposite sides in the diagram, e.g., the steps labeled with $\alpha$ and $\gamma(\beta$ and $\delta)$ in Fig. 2a and Fig. 2b, are due to applications of the same rule. Hence $\alpha=\gamma$ and $\beta=\delta$ in Fig. 2a and Fig. 2b, which shows the result.

Inspired by [11] we propose a labeling based on relative termination.

Lemma 8 Let $\mathcal{R}$ be a TRS and $\ell_{r t}(s \rightarrow t)=s$. Then $\ell_{r t}^{\mathcal{S}}=\left(\ell_{r t}, \rightarrow_{\mathcal{R}}^{*}, \rightarrow_{\mathcal{S} / \mathcal{R}}^{+}\right)$is an L-labeling for $\mathcal{R}$, provided $\rightarrow_{\mathcal{S}} \subseteq \rightarrow_{\mathcal{R}}$ and $\mathcal{S} / \mathcal{R}$ is terminating.

Proof Let $\geqslant=\rightarrow_{\mathcal{R}}^{*}$ and $>=\rightarrow_{\mathcal{S} / \mathcal{R}}^{+}$. First we show that $\left(\ell_{\mathrm{rt}}, \geqslant,>\right)$ is a labeling. By definition of relative rewriting, $\geqslant$ and $>$ are compatible and $>$ is well-founded by the termination assumption of $\mathcal{S} / \mathcal{R}$. Since rewriting is closed under contexts and substitutions, $\ell_{\mathrm{rt}}^{\mathcal{S}}$ is monotone and stable and hence a labeling. Next we show the properties demanded in Definition 5. The assumption $\rightarrow_{\mathcal{S}} \subseteq \rightarrow_{\mathcal{R}}$ yields $>\subseteq \geqslant$. Combining $\alpha=s=\beta, \gamma=u$, and $\delta=t$ with $s \rightarrow_{\mathcal{R}} t$ and $s \rightarrow_{\mathcal{R}} u$ yields $\alpha=\beta \geqslant \gamma, \delta$ for local peaks according to (parallel) and (variable-linear) in Fig. 2a and Fig. 2b.

The L-labeling from the previous lemma allows to establish a decrease with respect to some steps of $\mathcal{R}$. The next lemma allows to combine L-labelings. Let $\ell_{1}: \Lambda_{\mathcal{R}} \rightarrow W_{1}$ and $\ell_{2}: \Lambda_{\mathcal{R}} \rightarrow W_{2}$. Then $\left(\ell_{1}, \geqslant_{1},>_{1}\right) \times\left(\ell_{2}, \geqslant_{2},>_{2}\right)$ is defined as $\left(\ell_{1} \times \ell_{2}, \geqslant_{12},>_{12}\right)$ where $\ell_{1} \times \ell_{2}: \Lambda_{\mathcal{R}} \rightarrow W_{1} \times W_{2}$ with $\left(\ell_{1} \times \ell_{2}\right)(\Gamma)=\left(\ell_{1}(\Gamma), \ell_{2}(\Gamma)\right)$. Furthermore $\left(x_{1}, x_{2}\right) \geqslant 12$ $\left(y_{1}, y_{2}\right)$ if and only if $x_{1}>_{1} y_{1}$ or $x_{1} \geqslant_{1} y_{1}$ and $x_{2} \geqslant_{2} y_{2}$ and $\left(x_{1}, x_{2}\right)>_{12}\left(y_{1}, y_{2}\right)$ if and only if $x_{1}>_{1} y_{1}$ or $x_{1} \geqslant_{1} y_{1}$ and $x_{2}>_{2} y_{2}$. 
Lemma 9 Let $\ell_{1}$ and $\ell_{2}$ be L-labelings. Then $\ell_{1} \times \ell_{2}$ is an L-labeling.

Proof First we show that $\ell_{1} \times \ell_{2}$ is monotone and stable whenever $\ell_{1}$ and $\ell_{2}$ are labelings. Indeed if $\left(\ell_{1} \times \ell_{2}\right)(\Gamma) \geqslant\left(\ell_{1} \times \ell_{2}\right)(\Delta)$ then $\ell_{1}(\Gamma)>\ell_{1}(\Delta)$ or $\ell_{1}(\Gamma) \geqslant \ell_{1}(\Delta)$ and $\ell_{2}(\Gamma) \geqslant$ $\ell_{2}(\Delta)$, which for all contexts $C$ and substitutions $\sigma$ implies $\ell_{1}(C[\Gamma \sigma])>\ell_{1}(C[\Delta \sigma])$ or $\ell_{1}(C[\Gamma \sigma]) \geqslant \ell_{1}(C[\Delta \sigma])$ and $\ell_{2}(C[\Gamma \sigma]) \geqslant \ell_{2}(C[\Delta \sigma])$ by stability and monotonicity of $\ell_{1}$ and $\ell_{2}$, which is equivalent to $\left(\ell_{1} \times \ell_{2}\right)(C[\Gamma \sigma]) \geqslant\left(\ell_{1} \times \ell_{2}\right)(C[\Delta \sigma])$. Showing stability and monotonicity of $>$ is similar. Since the lexicographic product satisfies $>_{12} \subseteq \geqslant_{12}$ if $\ell_{1}$ and $\ell_{2}$ are labelings we conclude that $\ell_{1} \times \ell_{2}$ is a labeling.

Next we show that $\ell_{1} \times \ell_{2}$ satisfies the requirements of Definition 5. If $\ell_{1}$ and $\ell_{2}$ are Llabelings then the diagram of Fig. 2b has the shape as in Fig. 3a and Fig. 3b, respectively. It is easy to see that the lexicographic combination is again an L-labeling (cf. Fig. 3c).

\subsection{Left-Linear TRSs}

For left-linear TRSs the notion of an LL-labeling is introduced. The following definition exploits that Fig. 2b is an instance of Fig. 2c.

Definition 10 A labeling $\ell$ for a TRS $\mathcal{R}$ is an LL-labeling (for $\mathcal{R}$ ) if

1. in Fig. $2 \mathrm{a}, \alpha \geqslant \gamma$ and $\beta \geqslant \delta$,

2. $\quad$ in Fig. 2c, $\alpha \geqslant \bar{\gamma}$ and $\beta \geqslant \delta$ for all permutations of the rewrite steps of $u \Vdash v$, where $\alpha \geqslant \bar{\gamma}$ means $\alpha \geqslant \gamma_{i}$ for $1 \leqslant i \leqslant n$, and

3. in Fig. 2c, $\alpha>\bar{\gamma}$ for some permutation of the rewrite steps of $u \rightarrow v$, where $\alpha>\bar{\gamma}$ means $\alpha \geqslant \gamma_{1}$ and $\alpha>\gamma_{i}$ for $2 \leqslant i \leqslant n$.

A labeling $\ell$ is a weak LL-labeling if the first two conditions are satisfied.

We strengthened the definition of (weak) LL-labelings from [31]. All labelings proposed in [31] satisfy the stronger conditions. Considering all permutations in condition 2 of Definition 10 is necessary to ensure that the lexicographic combination of two weak LL-labelings again is a weak LL-labeling (cf. Lemma 14). Furthermore, this condition facilitates their use for parallel rewriting (Section 4).

Remark 11 The L-labelings presented so far (cf. Lemmata 7 and 8) are weak LL-labelings.

The next theorem states that LL-labelings allow to show confluence of left-linear TRSs.

Theorem 12 Let $\mathcal{R}$ be a left-linear TRS. If the critical peaks of $\mathcal{R}$ are LL-decreasing then $\mathcal{R}$ is confluent.

Proof By assumption the critical peaks of $\mathcal{R}$ are decreasing for some LL-labeling $\ell$. We establish confluence of $\mathcal{R}$ by Theorem 3, i.e., we show decreasingness of the ARS $\left\langle\mathcal{T}(\mathcal{F}, \mathcal{V}), \rightarrow_{\mathcal{R}}\right\rangle$ by labeling rewrite steps according to $\ell$. By definition of an LL-labeling local peaks according to (parallel) and (variable-left-linear) are decreasing. The reasoning for local peaks according to (critical overlap) is the same as in the proof of Theorem 6.

The rule labeling from Lemma 7 is a weak LL-labeling but not an LL-labeling since in Fig. 2c we have $\alpha=\gamma_{i}$ for $1 \leqslant i \leqslant n$ which does not satisfy $\alpha>\bar{\gamma}$ if $n>1$. (See also [11, Example 9].) We return to this problem and propose two solutions (in Sections 3.2.4 
and 4) after presenting simpler (weak) LL-labelings based on measuring duplicating steps (Section 3.2.1), the context above the contracted redex (Section 3.2.2), and the contracted redex (Section 3.2.3).

\subsubsection{Measuring Duplicating Steps}

The L-labeling from Lemma 8 can be adapted to an LL-labeling.

Lemma 13 Let $\mathcal{R}$ be a TRS. Then $\ell_{r t}^{\mathcal{R}_{d}}$ is an LL-labeling, provided $\mathcal{R}_{d} / \mathcal{R}_{n d}$ is terminating.

Proof By Theorem 2 the relative TRS $\mathcal{R}_{d} / \mathcal{R}_{n d}$ is terminating if and only if $\mathcal{R}_{d} / \mathcal{R}$ is terminating. Hence $\left(\ell_{\mathrm{rt}}^{\mathcal{R}_{d}}, \geqslant,>\right)$ is a labeling by Lemma 8 . Here $\geqslant=\rightarrow_{\mathcal{R}}^{*}$ and $>=\rightarrow_{\mathcal{R}_{d} / \mathcal{R}}^{+}$. Since $\ell_{\mathrm{rt}}(s \rightarrow t)=s$, we have $\alpha=\beta$ in Fig. 2a and Fig. 2c. We have $>\subseteq \geqslant$. Hence $\alpha \geqslant \gamma$ and $\alpha \geqslant \delta$ in Fig. 2a and, if $l_{2} \rightarrow r_{2}$ in local peak (1) is linear, also in Fig. 2c as $\bar{\gamma}$ is empty or $\bar{\gamma}=\gamma$ in this case. If $l_{2} \rightarrow r_{2}$ is not linear then it must be duplicating and hence $\alpha>\gamma_{i}$ for $1 \leqslant i \leqslant n$. Because $\alpha \geqslant \delta, \ell_{\mathrm{rt}}^{\mathcal{R}_{d}}$ is an LL-labeling for $\mathcal{R}$.

To combine the previous lemma with the rule labeling we study how different labelings can be combined.

Lemma 14 Let $\ell_{1}$ be an LL-labeling and let $\ell_{2}$ be a weak LL-labeling. Then $\ell_{1} \times \ell_{2}$ and $\ell_{2} \times \ell_{1}$ are LL-labelings.

Proof By the proof of Lemma $9 \ell_{1} \times \ell_{2}$ and $\ell_{2} \times \ell_{1}$ are labelings. The only interesting case of (variable-left-linear) is when $l_{2} \rightarrow r_{2}$ in local peak (1) is non-linear, i.e., $\bar{\gamma}$ contains more than one element. First we show that $\ell_{1} \times \ell_{2}$ is an LL-labeling. Here labels according to $\ell_{1}$ are suffixed with the subscript 1 and similarly for $\ell_{2}$. Recall Fig. 2c. Let us first deal with Definition 10(2). We have $\alpha_{1} \geqslant \bar{\gamma}_{1}, \beta_{1} \geqslant \delta_{1}, \alpha_{2} \geqslant \bar{\gamma}_{2}$ and $\beta_{2} \geqslant \delta_{2}$, which yields $\left(\beta_{1}, \beta_{2}\right) \geqslant$ $\left(\delta_{1}, \delta_{2}\right),\left(\alpha_{1}, \alpha_{2}\right) \geqslant\left(\gamma_{1 i}, \gamma_{2 i}\right)$ for all $1 \leqslant i \leqslant n$, by the definition of the lexicographic product. Next we consider Definition 10(3). By assumption we have $\alpha_{1}>\bar{\gamma}_{1}$, and $\alpha_{2} \geqslant \bar{\gamma}_{2}$, which yields the desired $\left(\alpha_{1}, \alpha_{2}\right) \geqslant\left(\gamma_{11}, \gamma_{21}\right),\left(\alpha_{1}, \alpha_{2}\right)>\left(\gamma_{1 i}, \gamma_{2 i}\right)$ for $2 \leqslant i \leqslant n$. In the proof for $\ell_{2} \times \ell_{1}$ the assumptions yield $\left(\beta_{2}, \beta_{1}\right) \geqslant\left(\delta_{2}, \delta_{1}\right)$ and $\left(\alpha_{2}, \alpha_{1}\right) \geqslant\left(\gamma_{2 i}, \gamma_{1 i}\right)$ for $1 \leqslant i \leqslant n$ for Definition 10(2) and additionally $\left(\alpha_{2}, \alpha_{1}\right)>\left(\gamma_{2 i}, \gamma_{1 i}\right)$ for $2 \leqslant i \leqslant n$ for Definition 10(3).

Remark 15 If $\ell_{1}$ and $\ell_{2}$ are weak LL-labelings then so are $\ell_{1} \times \ell_{2}$ and $\ell_{2} \times \ell_{1}$. Furthermore, LL-labelings are also weak LL-labelings by definition. In particular LL-labelings can be composed lexicographically.

From Theorem 12 and Lemmata 13 and 14 we obtain the following result.

Corollary 16 Let $\mathcal{R}$ be a left-linear TRS. If $\mathcal{R}_{d} / \mathcal{R}_{n d}$ is terminating and all critical peaks of $\mathcal{R}$ are weakly $L L$-decreasing then $\mathcal{R}$ is confluent.

Proof By Lemma $13 \ell_{\mathrm{rt}}^{\mathcal{R}_{d}}$ is an LL-labeling. By assumption the critical peaks of $\mathcal{R}$ are decreasing for some weak LL-labeling $\ell$. By Lemma 14 also $\ell_{\mathrm{rt}}^{\mathcal{R}_{d}} \times \ell$ is an LL-labeling. It remains to show decreasingness of the critical peaks for $\ell_{\mathrm{rt}}^{\mathcal{R}_{d}} \times \ell$. This is obvious since for terms $s, t, u$ with $s \rightarrow_{\mathcal{R}} t \rightarrow_{\mathcal{R}} u$ we have $\ell_{\mathrm{rt}}^{\mathcal{R}_{d}}(s \rightarrow t) \geqslant \ell_{\mathrm{rt}}^{\mathcal{R}_{d}}(t \rightarrow u)$. Hence 
decreasingness for $\ell$ implies decreasingness for $\ell_{\mathrm{rt}}^{\mathcal{R}_{d}} \times \ell$. Confluence of $\mathcal{R}$ follows from Theorem 12.

We revisit the example from the introduction.

Example 17 Recall the TRS $\mathcal{R}$ from Example 1. The polynomial interpretation

$$
+_{\mathbb{N}}(x, y)=x+y \quad \mathbf{s}_{\mathbb{N}}(x)=x+1 \quad \times_{\mathbb{N}}(x, y)=x^{2}+x y+y^{2} \quad \operatorname{sq}_{\mathbb{N}}(x)=3 x^{2}+1
$$

shows termination of $\mathcal{R}_{d} / \mathcal{R}_{n d}$. It is easy to check that $\ell_{\mathrm{rl}}^{i}$ with $i(3)=i(6)=2, i(4)=$ $i(10)=1$, and $i(l \rightarrow r)=0$ for all other rules $l \rightarrow r \in \mathcal{R}$ establishes decreasingness of the 34 critical peaks. We consider two selected critical peaks (where the applied rewrite rule is indicated above the arrow in parentheses). The peaks

$$
\begin{aligned}
& t_{1}=x+((y+z)+w) \underset{0}{\stackrel{(1)}{\leftarrow}} x+(y+(z+w)) \underset{0}{\stackrel{(1)}{\rightarrow}}(x+y)+(z+w)=u_{1} \\
& t_{2}=\mathbf{s}(x) \times \mathbf{s}(x) \underset{2}{\stackrel{(3)}{\leftarrow}} \mathrm{sq}(\mathbf{s}(x)) \underset{1}{\stackrel{(4)}{\longrightarrow}}(x \times x)+\mathbf{s}(x+x)=u_{2}
\end{aligned}
$$

can be joined decreasingly as follows:

$$
\begin{aligned}
t_{1} & \stackrel{(2)}{\rightarrow} x+(y+(z+w)) \\
t_{2} & \underset{0}{\stackrel{(10)}{\rightarrow}}(x \times \mathrm{s}(x))+\mathrm{s}(x) \underset{0}{\stackrel{(9)}{\leftrightarrows}} u_{1} \\
& \stackrel{(8)}{\rightarrow} x+(\mathrm{s}(x \times x)+x) \underset{0}{\stackrel{(2)}{\leftarrow}}(x+\mathrm{s}(x \times x))+x \underset{0}{\stackrel{(5)}{\leftarrow}}(\mathrm{s}(x \times x)+x)+x \\
& \stackrel{(1)}{\leftarrow} \mathrm{s}(x \times x)+(x+x) \underset{0}{\stackrel{(8)}{\leftarrow}} u_{2}
\end{aligned}
$$

The next example is concise and constitutes a minimal example to familiarize the reader with Corollary 16.

Example 18 Consider the TRS $\mathcal{R}$ consisting of the three rules

$$
1: \mathrm{b} \rightarrow \mathrm{a} \quad 2: \mathrm{a} \rightarrow \mathrm{b} \quad 3: \mathrm{f}(\mathrm{g}(x, \mathrm{a})) \rightarrow \mathrm{g}(\mathrm{f}(x), \mathrm{f}(x))
$$

We have $\mathcal{R}_{d}=\{3\}$ and $\mathcal{R}_{n d}=\{1,2\}$. Termination of $\mathcal{R}_{d} / \mathcal{R}_{n d}$ can be established by LPO with precedence $\mathrm{a} \sim \mathrm{b}$ and $\mathrm{f}>\mathrm{g}$. The rule labeling that takes the rule numbers as labels shows the only critical peak decreasing, i.e., $\mathrm{f}(\mathrm{g}(x, \mathrm{~b})){ }_{2} \leftarrow \mathrm{f}(\mathrm{g}(x, \mathrm{a})) \rightarrow_{3} \mathrm{~g}(\mathrm{f}(x), \mathrm{f}(x))$ and $\mathrm{f}(\mathrm{g}(x, \mathrm{~b})) \rightarrow_{1} \mathrm{f}(\mathrm{g}(x, \mathrm{a})) \rightarrow_{3} \mathrm{~g}(\mathrm{f}(x), \mathrm{f}(x))$. Hence we obtain the confluence of $\mathcal{R}$ by Corollary 16.

Remark 19 Using $\ell_{\mathrm{rl}}^{i}(\cdot)=0$ as weak LL-labeling, Corollary 16 gives a condition (termination of $\mathcal{R}_{d} / \mathcal{R}_{n d}$ ) such that $t \rightarrow{ }^{=} u$ or $u \rightarrow^{=} t$ for all critical pairs $t \leftarrow \rtimes \rightarrow u$ implies confluence of a left-linear TRS $\mathcal{R}$. This partially answers one question in the RTA list of open problems \#13. ${ }^{2}$

\footnotetext{
${ }^{2}$ http://www.cs.tau.ac.il/ nachum/rtaloop/problems/13.html
} 


\subsubsection{Measuring the context above the contracted redex}

In [17, Example 20] van Oostrom suggests to count function symbols above the contracted redex, demands that this measurement decreases for variables that are duplicated, and combines this with the rule labeling. Consequently local peaks according to Fig. $2 \mathrm{c}$ are decreasing. Below we exploit this idea but incorporate the following beneficial generalizations. First, we do not restrict to counting function symbols (which has been adopted and extended by Aoto in [1]) but represent the constraints as a relative termination problem. This abstract formulation allows to strictly subsume the criteria from [1, 17] (see Section 5) because more advanced techniques than counting symbols can be applied for proving termination. Additionally, our setting also allows to weaken these constraints significantly (cf. Lemma 27).

The next example motivates the need for an LL-labeling that does not require termination of $\mathcal{R}_{d} / \mathcal{R}_{n d}$.

Example 20 Consider the TRS $\mathcal{R}$ consisting of the six rules

$$
\begin{aligned}
& \mathrm{f}(\mathrm{h}(x)) \rightarrow \mathrm{h}(\mathrm{g}(\mathrm{f}(x), x, \mathrm{f}(\mathrm{h}(\mathrm{a})))) \quad \mathrm{f}(x) \rightarrow \mathrm{a} \quad \mathrm{a} \rightarrow \mathrm{b} \\
& \mathrm{h}(x) \rightarrow \mathrm{c} \quad \mathrm{b} \rightarrow \perp \quad \mathrm{c} \rightarrow \perp
\end{aligned}
$$

Since the duplicating rule admits an infinite sequence, Corollary 16 cannot succeed.

In the sequel we let $\mathcal{G}$ be the signature consisting of unary function symbols $f_{1}, \ldots, f_{n}$ for every $n$-ary function symbol $f \in \mathcal{F}$.

Definition 21 Let $x \in \mathcal{V}$. We define a partial mapping $\star$ from terms in the original signature and positions $\mathcal{T}(\mathcal{F}, \mathcal{V}) \times \mathbb{N}_{+}^{*}$ to terms in $\mathcal{T}(\mathcal{G}, \mathcal{V})$ as follows:

$$
\star\left(f\left(t_{1}, \ldots, t_{n}\right), p\right)= \begin{cases}f_{i}\left(\star\left(t_{i}, q\right)\right) & \text { if } p=i q \\ x & \text { if } p=\epsilon\end{cases}
$$

For a TRS $\mathcal{R}$ we abbreviate $\mathcal{R}_{>}^{\star} / \mathcal{R}_{=}^{\star}$ by $\star(\mathcal{R})$. Here, for $\gtrsim \in\{>,=\}, \mathcal{R}_{>}^{\star}$ consists of all rules $\star(l, p) \rightarrow \star(r, q)$ such that $l \rightarrow r \in \mathcal{R},\left.l\right|_{p}=\left.r\right|_{q}=y \in \mathcal{V}$, and $|r|_{y} \stackrel{\gtrsim}{\gtrsim} 1$.

The next example illustrates the transformation $\star(\cdot)$.

Example 22 Consider the TRS $\mathcal{R}$ from Example 20. The relative TRS $\star(\mathcal{R})=\mathcal{R}_{>}^{\star} / \mathcal{R}_{=}^{\star}$ consists of the TRS $\mathcal{R}_{>}^{\star}$ with rules

$$
\mathrm{f}_{1}\left(\mathrm{~h}_{1}(x)\right) \rightarrow \mathrm{h}_{1}\left(\mathrm{~g}_{1}\left(\mathrm{f}_{1}(x)\right)\right) \quad \mathrm{f}_{1}\left(\mathrm{~h}_{1}(x)\right) \rightarrow \mathrm{h}_{1}\left(\mathrm{~g}_{2}(x)\right)
$$

and the TRS $\mathcal{R}_{=}^{\star}$ which is empty.

Due to the next lemma a termination proof of $\star(\mathcal{R})$ yields an LL-labeling.

Lemma 23 Let $\mathcal{R}$ be a TRS and $\ell_{\star}\left(s \rightarrow^{\pi} t\right)=\star\left(s, p_{\pi}\right)$. Then $\left(\ell_{\star}, \geqslant,>\right)$ is an LL-labeling, provided $(\geqslant,>)$ is a monotone reduction pair, $\mathcal{R}_{>}^{\star} \subseteq>$, and $\mathcal{R}_{>}^{\star} \cup \mathcal{R}_{=}^{\star} \subseteq \geqslant$.

Proof Because $(\geqslant,>)$ is a monotone reduction pair, $\left(\ell_{\star}, \geqslant,>\right)$ is a labeling for $\mathcal{R}$. Note that monotonicity and stability are with respect to the signature $\mathcal{G}$. To see that the constraints of Definition 10 are satisfied we argue as follows. For Fig. 2a we have $\alpha=\gamma$ and $\beta=\delta$ because the steps drawn at opposing sides in the diagram take place at the same positions 
and the function symbols above these positions stay the same. Next we consider Fig. 2b, i.e., the right-linear case. Recall the local peak (1). Again we have $\beta=\delta$ because $q<p$. To see $\alpha \geqslant \gamma$ consider the step $s \rightarrow^{q, l_{2} \rightarrow r_{2}} u$ and let $q^{\prime}$ be the unique position in $\mathcal{P o s} \mathcal{V}\left(l_{2}\right)$ such that $q q^{\prime} r=p$ with $x=\left.l_{2}\right|_{q^{\prime}}$ for some position $r$. If $\left|r_{2}\right|_{x}=0$ then there is no step and we are done. Otherwise let $q^{\prime \prime}$ be the position in $r_{2}$ with $\left|r_{2}\right|_{q^{\prime \prime}}=x$. By construction $\mathcal{R}_{=}^{\star}$ contains the rule $\star\left(l_{2}, q^{\prime}\right) \rightarrow \star\left(r_{2}, q^{\prime \prime}\right)$. Combining the assumption $\mathcal{R}_{=}^{\star} \subseteq \geqslant$ with monotonicity and stability of $\ell_{\star}$ yields $\star(s, p) \geqslant \star\left(u, q q^{\prime \prime} r\right)$, i.e., $\alpha \geqslant \gamma$. Next we consider Fig. 2c for the duplicating case. Recall the local peak (1). Again we have $\beta=\delta$ because $q<p$. To see $\alpha>\bar{\gamma}$ (for any permutation of the steps) consider the step $s \rightarrow^{q, l_{2} \rightarrow r_{2}} u$ and let $q^{\prime}$ be the unique position in $\operatorname{Pos}_{\mathcal{V}}\left(l_{2}\right)$ such that $q q^{\prime} r=p$ for some position $r$. Let $x=\left.l_{2}\right|_{q^{\prime}}$ and $Q=\left\{q_{1}^{\prime}, \ldots, q_{n}^{\prime}\right\}$ with $\left.r_{2}\right|_{q_{i}^{\prime}}=x$. Then $P=\left\{q q_{i}^{\prime} r \mid q_{i}^{\prime} \in Q\right\}$ is the set of descendants of $p$. By construction $\mathcal{R}_{>}^{\star}$ contains all rules $\star\left(l_{2}, q^{\prime}\right) \rightarrow \star\left(r_{2}, q_{i}^{\prime}\right)$ for $1 \leqslant i \leqslant n$. Combining the assumption $\mathcal{R}_{>}^{\star} \subseteq>$ with monotonicity and stability of $\ell_{\star}$ yields $\star(s, p)>\star\left(u, p_{i}^{\prime}\right)$ for $p_{i}^{\prime} \in P$. Since $u \rightarrow^{P} v$ we obtain $\alpha>\gamma_{i}$ for $1 \leqslant i \leqslant n$ and hence the desired $\alpha>\bar{\gamma}$.

Remark 24 It is also possible to formulate Lemma 23 as a relative termination criterion without the use of a monotone reduction pair. However, the monotone reduction pair may admit more labels to be comparable (in the critical diagrams) because of the inclusions $\mathcal{R}_{>}^{\star} \subseteq>$ and $\mathcal{R}_{>}^{\star} \cup \mathcal{R}_{=}^{\star} \subseteq \geqslant$.

From Lemma 23 we obtain the following corollary.

Corollary 25 Let $\mathcal{R}$ be a left-linear TRS and let $\ell$ be a weak LL-labeling. Let $\ell_{\star} \ell$ denote $\ell \times \ell_{\star}$ or $\ell_{\star} \times \ell$. Let $(\geqslant,>)$ be a monotone reduction pair showing termination of $\star(\mathcal{R})$. If the critical peaks of $\mathcal{R}$ are decreasing for $\ell_{\star} \ell$ then $\mathcal{R}$ is confluent.

Proof The function $\ell_{\star}$ is an LL-labeling by Lemma 23. Lemma 14 yields that $\ell_{\star} \ell$ is an LL-labeling. By assumption the critical peaks are decreasing for $\ell_{\star} \ell$ and hence Theorem 12 yields the confluence of $\mathcal{R}$.

The next example illustrates the use of Corollary 25 .

Example 26 We show confluence of the TRS $\mathcal{R}$ from Example 20. Termination of $\star(\mathcal{R})$ (cf. Example 22) is easily shown, e.g., the polynomial interpretation

$$
\mathrm{f}_{1 \mathbb{N}}(x)=2 x \quad \mathrm{~g}_{1 \mathbb{N}}(x)=\mathrm{g}_{2 \mathbb{N}}(x)=x \quad \mathrm{~h}_{1 \mathbb{N}}(x)=x+1
$$

orients both rules in $\mathcal{R}_{>}^{\star}$ strictly. To show decreasingness of the three critical peaks (two of which are symmetric) we use the labeling $\ell_{\star} \times \ell_{\mathrm{rl}}^{i}$ with $i(\mathrm{f}(\mathrm{h}(x)) \rightarrow \mathrm{h}(\mathrm{g}(\mathrm{f}(x), x, \mathrm{f}(\mathrm{h}(\mathrm{a})))))=1$ and all other rules receive label 0 . For the moment we label a step $s \rightarrow^{\pi} t$ with the interpretation of $\star\left(s, p_{\pi}\right)$. E.g., a step $\mathrm{f}(\mathrm{h}(\mathrm{b})) \rightarrow \mathrm{f}(\mathrm{h}(\perp))$ is labeled $2 x+2$ since $\star(f(h(b)), 11)=\mathrm{f}_{1}\left(\mathrm{~h}_{1}(x)\right)$ and $\left[\mathrm{f}_{1}\left(\mathrm{~h}_{1}(x)\right)\right]_{\mathbb{N}}=2 x+2$. The critical peak $\mathrm{h}(\mathrm{g}(\mathrm{f}(x), x, \mathrm{f}(\mathrm{h}(\mathrm{a}))))_{x, 1} \leftarrow \mathrm{f}(\mathrm{h}(x)) \rightarrow_{x, 0}$ a is closed decreasingly by

$$
\mathrm{h}(\mathrm{g}(\mathrm{f}(x), x, \mathrm{f}(\mathrm{h}(\mathrm{a})))) \underset{x, 0}{\longrightarrow} \mathrm{c} \underset{x, 0}{\longrightarrow} \perp \underset{x, 0}{\longleftarrow} \mathrm{b} \underset{x, 0}{\longleftarrow} \mathrm{a}
$$

and the critical peak $\mathrm{h}(\mathrm{g}(\mathrm{f}(x), x, \mathrm{f}(\mathrm{h}(\mathrm{a}))))_{x, 1} \leftarrow \mathrm{f}(\mathrm{h}(x)) \rightarrow_{2 x, 0} \mathrm{f}(\mathrm{c})$ is closed decreasingly by

$$
\mathrm{h}(\mathrm{g}(\mathrm{f}(x), x, \mathrm{f}(\mathrm{h}(\mathrm{a})))) \underset{x, 0}{\longrightarrow} \mathrm{c} \underset{x, 0}{\longrightarrow} \perp \underset{x, 0}{\longleftarrow} \mathrm{b} \underset{x, 0}{\longleftarrow} \mathrm{a} \underset{x, 0}{\longleftarrow} \mathrm{f}(\mathrm{c})
$$

which allows to prove confluence of $\mathcal{R}$ by Corollary 25 . 
By definition of $\alpha>\bar{\gamma}$ (cf. Definition 10) we observe that the definition of $\star(\mathcal{R})$ can be relaxed. If $l_{2} \rightarrow r_{2}$ with $\left.l_{2}\right|_{q^{\prime}}=x \in \mathcal{V}$ and $\left\{q_{1}^{\prime}, \ldots, q_{n}^{\prime}\right\}$ are the positions of the variable $x$ in $r_{2}$ then it suffices if $n-1$ instances of $\star\left(l_{2}, q^{\prime}\right) \rightarrow \star\left(r_{2}, q_{i}^{\prime}\right)$ are put in $\mathcal{R}_{>}^{\star}$ while one $\star\left(l_{2}, q^{\prime}\right) \rightarrow \star\left(r_{2}, q_{j}^{\prime}\right)$ can be put in $\mathcal{R}_{=}^{\star}$ (since the steps labeled $\bar{\gamma}$ in Fig. 2c are at parallel positions we can choose the first closing step such that $\alpha \geqslant \gamma_{1}$ ). This improved version of $\star(\mathcal{R})$ is denoted by $\star(\mathcal{R})=\mathcal{R}_{>}^{\star \star} / \mathcal{R}_{=}^{\star \star}$. We obtain the following variant of Lemma 23 .

Lemma 27 Let $\mathcal{R}$ be a TRS. Then $\left(\ell_{\star}, \geqslant,>\right)$ is an LL-labeling, provided $(\geqslant,>)$ is a monotone reduction pair, $\mathcal{R}_{>}^{\star \star} \subseteq>$, and $\mathcal{R}_{>}^{\star \star} \cup \mathcal{R}_{=}^{\star \star} \subseteq \geqslant$.

Obviously any $\star(\mathcal{R})$ inherits termination from $\star(\mathcal{R})$. The next example shows that the reverse statement does not hold. In Section 6 we show how the intrinsic indeterminism of $\star(\mathcal{R})$ is eliminated in the implementation.

Example 28 Consider the TRS $\mathcal{R}$ from Example 1. The TRS $\mathcal{R}_{>}^{\star}$ consists of the rules

$$
\begin{array}{llc}
\mathrm{sq}_{1}(x) \rightarrow \times_{1}(x) & \mathrm{sq}_{1}\left(\mathrm{~s}_{1}(x)\right) \rightarrow+_{1}\left(\times_{1}(x)\right) & \times_{1}(x) \rightarrow+_{1}(x) \\
\mathrm{sq}_{1}(x) \rightarrow \times_{2}(x) & \mathrm{sq}_{1}\left(\mathrm{~s}_{1}(x)\right) \rightarrow+_{1}\left(\times_{2}(x)\right) & \dagger: \times_{1}(x) \rightarrow+_{2}\left(\times_{1}(x)\right) \\
& \mathrm{sq}_{1}\left(\mathrm{~s}_{1}(x)\right) \rightarrow+_{2}\left(\mathrm{~s}_{1}\left(+_{1}(x)\right)\right) & \dagger: \times_{2}(y) \rightarrow+_{1}\left(\times_{2}(y)\right) \\
& \mathrm{sq}_{1}\left(\mathrm{~s}_{1}(x)\right) \rightarrow+_{2}\left(\mathrm{~s}_{1}\left(+_{2}(x)\right)\right) & \times_{2}(y) \rightarrow+_{2}(y)
\end{array}
$$

while $\mathcal{R}_{=}^{\star}$ consists of the rules

$$
\begin{array}{rlrl}
+_{1}(x) & \rightarrow+_{1}\left(+_{1}(x)\right) & & +_{1}(x) \rightarrow+_{2}(x) \\
+_{2}\left(+_{1}(y)\right) & \rightarrow+_{1}\left(+_{2}(y)\right) & & +_{2}(y) \rightarrow+_{1}(y) \\
+_{2}\left(+_{2}(z)\right) & \rightarrow+_{2}(z) & \times_{1}(x) & \rightarrow \times_{2}(x) \\
+_{1}\left(+_{1}(x)\right) & \rightarrow+_{1}(x) & \times_{2}(y) \rightarrow \times_{1}(y) \\
+_{1}\left(+_{2}(y)\right) & \rightarrow+_{2}\left(+_{1}(y)\right) & +_{1}\left(\mathrm{~s}_{1}(x)\right) & \rightarrow+_{1}(x) \\
+_{2}(z) & \rightarrow+_{2}\left(+_{2}(z)\right) & +_{2}(y) & \rightarrow+_{2}\left(\mathrm{~s}_{1}(y)\right)
\end{array}
$$

$$
\begin{gathered}
+_{1}(x) \rightarrow+{ }_{1}\left(\mathrm{~s}_{1}(x)\right) \\
+_{2}\left(\mathrm{~s}_{1}(y)\right) \rightarrow+{ }_{2}(y) \\
\times_{2}\left(\mathrm{~s}_{1}(y)\right) \rightarrow+{ }_{2}\left(\times_{2}(y)\right) \\
\times \times_{1}\left(\mathrm{~s}_{1}(x)\right) \rightarrow+{ }_{1}\left(\times_{1}(x)\right)
\end{gathered}
$$

Let $\mathcal{R}_{\dagger}^{\star}$ denote the rules in $\mathcal{R}_{>}^{\star}$ marked with $\dagger$. Termination of $\star(\mathcal{R})$ cannot be established (because $\mathcal{R}_{\dagger}^{\star}$ is non-terminating) but we stress that moving these rules into $\mathcal{R}_{=}^{\star}$ yields a valid $\star(\mathcal{R})$ which can be proved terminating by the polynomial interpretation with

$$
\mathrm{sq}_{1 \mathbb{N}}(x)=x+2 \quad \times_{1 \mathbb{N}}(x)=\times_{2 \mathbb{N}}(x)=x+1
$$

that interprets the remaining function symbols by the identity function. We remark that Corollary 25 with the labeling from Lemma 27 establishes confluence of $\mathcal{R}$. Since all reductions in the 34 joining sequences have only + above the redex and $+_{1 \mathbb{N}}(x)=+_{2 \mathbb{N}}(x)=x$, the $\ell_{\star}$ labeling attaches $x$ to any of these steps. The rule labeling that assigns $i(3)=i(6)=2, i(4)=i(10)=1$, and 0 to all other rules shows the 34 critical peaks decreasing.

\subsubsection{Measuring the contracted redex}

Instead of the labeling $\ell_{\star}$, which is based on the context above the contracted redex, one can also use the contracted redex itself for labeling.

Lemma 29 Let $\mathcal{R}$ be a TRS and $\ell_{\triangle}\left(s \rightarrow^{\pi} t\right)=\left.s\right|_{p_{\pi}}$. Then $\left(\ell_{\triangle}, \geqslant,>\right)$ is a weak LLlabeling, provided $(\geqslant,>)$ is a monotone reduction pair with $\mathcal{R} \subseteq \geqslant$. 
Proof Because $(\geqslant,>)$ is a monotone reduction pair, $\left(\ell_{\Delta}, \geqslant,>\right)$ is a labeling for $\mathcal{R}$. To see that the constraints of Definition 10 are satisfied we argue as follows. For Fig. 2a we have $\alpha=\gamma$ and $\beta=\delta$. For Fig. 2c we have $\alpha=\gamma_{1}=\cdots=\gamma_{n}$ (since the same redex is contracted) and $\beta \geqslant \delta$ by the assumption $\mathcal{R} \subseteq \geqslant$ and monotonicity and stability of $\geqslant$.

The following definition collects the constraints, such that variable overlaps can be made decreasing.

Definition 30 For a TRS $\mathcal{R}$ let $\mathcal{R}^{\triangle}=\left\{l \rightarrow x \mid l \rightarrow r \in \mathcal{R}\right.$ and $\left.|r|_{x}>1\right\}$.

Due to the next result a termination proof of $\mathcal{R}^{\triangle} / \mathcal{R}$ enables a weak LL-labeling to establish confluence.

Corollary 31 Let $\mathcal{R}$ be a left-linear TRS and let $\ell$ be a weak LL-labeling. Let $(\geqslant,>)$ be a simple monotone reduction pair showing termination of $\mathcal{R} \Delta / \mathcal{R}$. If the critical peaks of $\mathcal{R}$ are decreasing for $\ell_{\Delta} \times \ell$ then $\mathcal{R}$ is confluent.

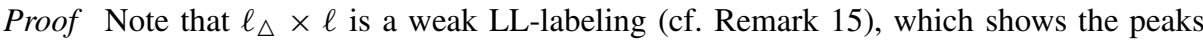
in Fig. $2 \mathrm{a}$ and Fig. $2 \mathrm{~b}$ decreasing. For the duplicating case of Fig. $2 \mathrm{c}$ we inspect the labels with regard to $\ell_{\triangle}$. Consider the local peak (1). Clearly, $\beta=l_{2} \sigma$ and $\alpha=l_{1} \sigma$. Since $\gamma_{i}=\alpha$, we want to establish $\beta>\alpha$. To this end let $q^{\prime} \in \mathcal{P}$ os $\mathcal{V}\left(l_{2}\right)$ such that $q q^{\prime} r=p$ and $x=\left.l_{2}\right|_{q^{\prime}}$. Note that $l_{2} \rightarrow x \in \mathcal{R}^{\triangle}$ because we are in the duplicating case. Hence the relative termination assumption gives $l_{2}>x$, and $l_{2} \sigma>x \sigma$ is obtained by stability. Now as $\left.x \sigma\right|_{r}=l_{1} \sigma$ the desired $\beta>\alpha$ follows from simplicity of the reduction pair since $l_{2} \sigma>x \sigma \geqslant l_{1} \sigma$. Combining $\ell_{\triangle}$ lexicographically with a weak LL-labeling $\ell$ into $\ell_{\triangle} \times \ell$ maintains decreasingness.

Remark 32 Note that the labeling $\ell_{\Delta} \times \ell$ from Corollary 31 is not an LL-labeling. The point is that there are multiple ways of ensuring decreasingness of Fig. 2c. For LL-labelings, we use $\alpha>\bar{\gamma}$, while in Corollary 31, $\beta>\gamma_{i}$ for $1 \leqslant i \leqslant n$ does the job. This is also the reason why $\ell \times \ell_{\triangle}$ cannot be used in Corollary 31. Consider the TRS with the rules $1: \mathrm{f}(x) \rightarrow \mathrm{g}(x, x)$ and $2: \mathrm{a} \rightarrow \mathrm{b}$. Let $\ell_{\mathrm{rl}}$ be the rule labeling attaching the rule numbers as labels. Then the variable overlap is not decreasing for $\ell_{\mathrm{rl}} \times \ell_{\Delta}$.

We demonstrate Corollary 31 on the TRS from Example 18.

Example 33 Consider the TRS from Example 18. The polynomial interpretation

$$
\mathrm{g}_{\mathbb{N}}(x, y)=2 x+2 y+1 \quad \mathrm{a}_{\mathbb{N}}=\mathrm{b}_{\mathbb{N}}=0 \quad \mathrm{f}_{\mathbb{N}}(x)=x^{2}
$$

establishes relative termination of $\{\mathrm{f}(\mathrm{g}(x, \mathrm{a})) \rightarrow x\} / \mathcal{R}$ and shows the critical peak decreasing when labeling steps with the pair obtained by the interpretation of the redex and the rule labeling, i.e., $t=\mathrm{f}(\mathrm{g}(x, \mathrm{~b})) 0_{0,2} \leftarrow \mathrm{f}(\mathrm{g}(x, \mathrm{a})) \rightarrow_{(2 x+1)^{2}, 3} \mathrm{~g}(\mathrm{f}(x), \mathrm{f}(x))=u$ for the peak and $t \rightarrow_{0,1} \mathrm{f}(\mathrm{g}(x, \mathrm{a})) \rightarrow_{(2 x+1)^{2}, 3} u$ for the join.

\subsubsection{Exploiting Persistence}

In this section we show how to exploit persistence of confluence [3, 7] to enhance the applicability of L-labelings to certain duplicating left-linear TRSs. Compared to 
Sections 3.2.1-3.2.3, where variable overlaps were closed decreasingly by a relative termination criterion, here persistence arguments are employed to avoid reasoning about variable overlaps at duplicating variable positions at all. To this end we recall order-sorted TRSs.

Definition 34 Let $S$ be a set of sorts equipped with a partial order $\leq$. A signature $\mathcal{F}$ and a set of variables $\mathcal{V}$ are $S$-sorted if every $n$-ary function symbol $f \in \mathcal{F}$ is equipped with a sort declaration $\alpha_{1} \times \cdots \times \alpha_{n} \rightarrow \alpha$ where $\alpha_{1}, \ldots, \alpha_{n}, \alpha \in S$ and every variable $x \in \mathcal{V}$ has exactly one sort $\alpha \in S$. We write $S(f)=\alpha, S(f, i)=\alpha_{i}$ for $1 \leqslant i \leqslant n$, and $S(x)=\alpha$, respectively. We let $\mathcal{V}_{\alpha}=\{x \in \mathcal{V} \mid S(x)=\alpha\}$ and require that $\mathcal{V}_{\alpha}$ is infinite for all $\alpha \in S$. The set of $S$-sorted terms, $\mathcal{T}_{S}(\mathcal{F}, \mathcal{V})$, is the union of the sets $\mathcal{T}_{\alpha}(\mathcal{F}, \mathcal{V})$ for $\alpha \in S$ that are inductively defined as follows: $\mathcal{V}_{\alpha} \subseteq \mathcal{T}_{\alpha}(\mathcal{F}, \mathcal{V})$ and $f\left(t_{1}, \ldots, t_{n}\right) \in \mathcal{T}_{\alpha}(\mathcal{F}, \mathcal{V})$ whenever $f \in \mathcal{F}$ has sort declaration $\alpha_{1} \times \cdots \times \alpha_{n} \rightarrow \alpha$ and $t_{i} \in \mathcal{T}_{\leq \alpha_{i}}(\mathcal{F}, \mathcal{V})$ for all $1 \leqslant i \leqslant n$. Here $\mathcal{T}_{\leq \alpha}(\mathcal{F}, \mathcal{V})$ is the union of all $\mathcal{T}_{\beta}(\mathcal{F}, \mathcal{V})$ for $\beta \leq \alpha$.

The notion of $S$-sorted terms properly extends many-sorted terms. Indeed, if we let $\leq$ be the identity relation then $\mathcal{T}_{\leq \alpha}(\mathcal{F}, \mathcal{V})=\mathcal{T}_{\alpha}(\mathcal{F}, \mathcal{V})$, which means that the $i$-th argument of $f$ in an $S$-sorted term must have sort $S(f, i)$.

Definition 35 We extend $S(\cdot)$ and $S(\cdot, \cdot)$ to $S$-sorted terms $t$ and non-root positions of $t$. If $t=f\left(t_{1}, \ldots, t_{n}\right)$ then $S(t)=S(f), S(t, i)=S(f, i)$, and $S(t, i p)=S\left(t_{i}, p\right)$ for $p \neq \epsilon$. If $t=x \in \mathcal{V}$ then $S(t)=S(x)$.

Example 36 Let $S=\{0,1,2\}$ with $0 \leq 1$ and consider the sort declarations $f: 1 \rightarrow 2$ and $x: 0$. Then $t=\mathrm{f}(x) \in \mathcal{T}_{S}(\{\mathrm{f}\},\{x\}), S(t)=2, S(t, 1)=1$, and $S\left(\left.t\right|_{1}\right)=0 \leq 1$.

One easily observes that $S(t, p)$ defines the maximal sort induced by the context $t[\square]_{p}$ : a term $t[u]_{p}$ is $S$-sorted if and only if $u \in \mathcal{T}_{\leq S(t, p)}(\mathcal{F}, \mathcal{V})$. Consequently, we have $S\left(\left.t\right|_{p}\right) \leq S(t, p)$ for all non-root positions $p$ of $t$.

We are particularly interested in the case where rewriting restricted to $S$-sorted terms coincides with ordinary rewriting with initial terms restricted to $S$-sorted ones. This property is captured by $S$-compatible TRSs.

Definition 37 A TRS $\mathcal{R}$ is $S$-compatible if for every rule $l \rightarrow r \in \mathcal{R}$ there exists a sort $\alpha \in S$ such that $l \in \mathcal{T}_{\alpha}(\mathcal{F}, \mathcal{V})$ and $r \in \mathcal{T}_{\leq \alpha}(\mathcal{F}, \mathcal{V})$, and $S(l, p)=S\left(\left.l\right|_{p}\right)$ for all $p \in$ $\operatorname{Pos}_{\mathcal{V}}(l)$.

The following lemma is well-known (e.g. [27]) and easy to prove.

Lemma 38 If $\mathcal{R}$ is $S$-compatible then $\mathcal{T}_{S}(\mathcal{F}, \mathcal{V})$ and $\mathcal{T}_{\leq \alpha}(\mathcal{F}, \mathcal{V})$ for every $\alpha \in S$ are closed under rewriting by $\mathcal{R}$.

The following result is a special case of [7, Theorem 6.2].

Theorem 39 An S-compatible left-linear TRS $\mathcal{R}$ is confluent on $\mathcal{T}(\mathcal{F}, \mathcal{V})$ if and only if it is confluent on $\mathcal{T}_{S}(\mathcal{F}, \mathcal{V})$.

Example 40 Consider the duplicating TRS $\mathcal{R}$ with rules

$$
1: \mathrm{f}(\mathrm{a}) \rightarrow \mathrm{f}(\mathrm{b}) \quad 2: \mathrm{f}(x) \rightarrow \mathrm{g}(\mathrm{f}(x), \mathrm{f}(x))
$$


Recall that L-labelings (in particular, rule labelings) that are not LL-labelings are not applicable to non-linear TRSs because the variable overlap diagram (Fig. 2c) is not decreasing. Let $S=\{0,1\}$ with the following sort declarations:

$$
x: 0 \quad \mathrm{a}: 0 \quad \mathrm{~b}: 0 \quad \mathrm{f}: 0 \rightarrow 1 \quad \mathrm{~g}: 1 \times 1 \rightarrow 1
$$

The TRS $\mathcal{R}$ is $S$-compatible and hence we may restrict rewriting to $S$-sorted terms without affecting confluence by Theorem 39. This has the beneficial effect that variable overlaps are ruled out. To see how, note that no subterms of sort 1 can appear inside terms of sort 0 . Consider the left-hand side $\mathrm{f}(x)$ of $\mathcal{R}$. We have $S(\mathrm{f}(x), 1)=0$, so that any term substituted for $x$ must have sort 0 . Further note that both left-hand sides have sort 1 . Consequently, no rule application may be nested below $\mathrm{f}(x) \rightarrow \mathrm{g}(\mathrm{f}(x), \mathrm{f}(x))$ and hence variable overlaps are ruled out. Therefore, we may use L-labelings to show confluence of $\mathcal{R}$ even though $\mathcal{R}$ is not linear, and in fact the rule labeling which takes the rule numbers as labels allows us to join the sole (modulo symmetry) critical peak $t=\mathrm{f}(\mathrm{b})_{1} \leftarrow \mathrm{f}(\mathrm{a}) \rightarrow_{2} \mathrm{~g}(\mathrm{f}(\mathrm{a}), \mathrm{f}(\mathrm{a}))=u$ decreasingly: $t \rightarrow_{2} \mathrm{~g}(\mathrm{f}(\mathrm{b}), \mathrm{f}(\mathrm{b}))_{1} \leftarrow \mathrm{g}(\mathrm{f}(\mathrm{b}), \mathrm{f}(\mathrm{a}))_{1} \leftarrow u$.

Formally, we define $\mathcal{T}_{\unlhd \alpha}(\mathcal{F}, \mathcal{V})=\left\{t \mid t \unlhd t^{\prime}\right.$ for some $\left.t^{\prime} \in \mathcal{T}_{\leq \alpha}(\mathcal{F}, \mathcal{V})\right\}$, to capture which terms may occur as subterms of terms of sort $\alpha$ or below.

Theorem 41 Let $\mathcal{R}$ be a left-linear $S$-compatible TRS such that the variable $\left.l\right|_{p}$ occurs at most once in $r$ whenever $l \rightarrow r \in \mathcal{R}$ and $l^{\prime} \rightarrow r^{\prime} \in \mathcal{R}$ with $l^{\prime} \in \mathcal{T}_{\unlhd S(l, p)}(\mathcal{F}, \mathcal{V})$ for some $p \in \mathcal{P o s}_{\mathcal{V}}(l)$. Then $\mathcal{R}$ is confluent if all its critical peaks are $L$-decreasing.

Proof By Theorem 39 we may restrict rewriting to $S$-sorted terms. The proof follows that of Theorem 6, except in the analysis of local peaks, where right-linearity of $\mathcal{R}$ is used, which is not among our assumptions. Instead, we argue as follows: Since $\mathcal{R}$ is left-linear, any local peak has the shape (parallel), (critical overlap), or (variable-left-linear). In the latter case, the step $s \rightarrow^{q, l^{\prime} \rightarrow r^{\prime}} t$ is nested below $s \rightarrow^{p, l \rightarrow r} u$, and it is easy to see that this implies $l^{\prime} \in \mathcal{T}_{\unlhd S\left(l, q^{\prime}\right)}(\mathcal{F}, \mathcal{V})$ for some variable position $q^{\prime}$ of $l$ such that $p q^{\prime} \leqslant q$. Consequently the variable $x=\left.l\right|_{q^{\prime}}$ occurs at most once in $r$ by assumption, and the parallel step (which contains one rewrite step for every occurrence of $x$ in $r$ ) is empty or a single step, resulting in a decreasing diagram.

As a refinement of Theorem 41, instead of ruling out duplicating (variable-left-linear) overlaps completely, we can also add additional constraints on the labeling for the remaining variable overlaps.

Definition 42 Let $\ell$ be a weak LL-labeling for an $S$-compatible TRS $\mathcal{R}$. We call $\ell$ persistent if whenever rules $l \rightarrow r, l^{\prime} \rightarrow r^{\prime} \in \mathcal{R}$ satisfy $l^{\prime} \in \mathcal{T}_{\triangleleft S(l, p)}(\mathcal{F}, \mathcal{V})$ for some $p \in \mathcal{P o s}_{\mathcal{V}}(l)$, either $|r|_{\left.l\right|_{p}} \leqslant 1$ or $\beta>\bar{\gamma}$ in Fig. 2c for all resulting variable overlaps with $l^{\prime} \rightarrow r^{\prime}$ below $l \rightarrow r$. We call $\mathcal{R}$ persistent LL-decreasing if there is a persistent, weak LL-labeling $\ell$ such that all critical peaks of $\mathcal{R}$ are decreasing with respect to $\ell$.

Theorem 43 Let $\mathcal{R}$ be a left-linear TRS. If the critical peaks of $\mathcal{R}$ are persistent LL-decreasing then $\mathcal{R}$ is confluent.

Proof The proof follows along the lines of the proof of Theorem 41. In the case of a duplicating variable-left-linear overlap, the additional constraints ensure that the resulting diagram is decreasing. 
Example 44 Suppose we extend the TRS from Example 40 with the rule a $\rightarrow$ b, using the same sorts:

$$
\text { 1: } \mathrm{f}(x) \rightarrow \mathrm{g}(\mathrm{f}(x), \mathrm{f}(x)) \quad 2: \mathrm{f}(\mathrm{a}) \rightarrow \mathrm{f}(\mathrm{b}) \quad 3: \mathrm{a} \rightarrow \mathrm{b}
$$

Theorem 41 is no longer applicable, because rule 3 may be nested below rule 1 , which is duplicating. However, by the preceding remark, any rule labeling with $\ell_{\mathrm{rl}}^{i}(1)>\ell_{\mathrm{rl}}^{i}(3)$ will make the corresponding variable overlaps decreasing.

Remark 45 Note that Theorem 43 does not subsume Theorem 41, because the former demands a weak LL-labeling whereas the latter requires only an L-labeling. If we were to restrict the L-labeling and weak LL-labeling conditions to those variable overlaps that are consistent with the sort declarations, then Theorem 43 would subsume Theorem 41 . We chose not to do so because all our labelings are weak LL-labelings.

The following example shows that considering order-sorted instead of many-sorted signatures is beneficial.

Example 46 Consider the duplicating TRS $\mathcal{R}$ given by the rules

$$
\text { 1: } \mathrm{h}(\mathrm{a}, \mathrm{a}) \rightarrow \mathrm{f}(\mathrm{a}) \quad 2: \mathrm{f}(\mathrm{a}) \rightarrow \mathrm{a} \quad 3: \mathrm{f}(x) \rightarrow \mathrm{h}(x, x)
$$

Furthermore, let $\mathcal{S}=\{0,1\}$ with $1>0$ and take the sort declarations

$$
\mathrm{h}: 0 \times 0 \rightarrow 1 \quad \mathrm{f}: 0 \rightarrow 1 \quad \mathrm{a}: 0
$$

Considering only $\mathcal{S}$-sorted terms, no rule can be nested below the duplicating rule $\mathrm{f}(x) \rightarrow$ $\mathrm{h}(x, x)$. Basically, there is one critical peak, $\mathrm{h}(\mathrm{a}, \mathrm{a})^{3} \leftarrow \mathrm{f}(\mathrm{a}) \rightarrow^{2}$ a, which is decreasingly joinable as $\mathrm{h}(\mathrm{a}, \mathrm{a}) \rightarrow^{1} \mathrm{f}(\mathrm{a}) \rightarrow^{2}$ a by the rule labeling (using rule numbers as labels), and confluence follows by Theorem 41 . Due to the rule $\mathrm{f}(\mathrm{a}) \rightarrow \mathrm{a}$, any many-sorted sort declaration for $\mathcal{R}$ must assign the same sorts to a and the argument and result types of $\mathrm{f}$. Therefore, $\mathrm{f}(x) \rightarrow \mathrm{h}(x, x)$ may be nested below itself, and Theorems 41 and 43 would fail in connection with the rule labeling.

\section{Labelings for Parallel Rewriting}

In this section, rather than labeling individual rewrite steps, we will label parallel rewrite steps instead. This is inspired by the parallel moves lemma, which says that any peak $t \ll s \boxplus u$ of two non-overlapping parallel rewrite steps can be joined in a diamond as $t \leftrightarrow * \leftrightarrow$, and diamonds are comparatively easy to label decreasingly, as we saw in Section 3.1.

The main problem is to label parallel steps such that variable overlaps are decreasing. The multiset of the single steps’ labels does not work since $\{\alpha\} \ngtr_{\text {mul }}\{\alpha, \ldots, \alpha\}$. Hence we use sets to label parallel steps which we denote by capital Greek letters. Sets of labels are ordered by the Hoare preorder of $(\geqslant,>)$, which we denote by $\left(\geqslant_{H},>_{H}\right)$ and is defined by

$$
\begin{array}{ll}
\Gamma>_{H} \Delta & \Longleftrightarrow \quad \Gamma \neq \varnothing \wedge \forall \beta \in \Delta \exists \alpha \in \Gamma(\alpha>\beta) \\
\Gamma \geqslant_{H} \Delta & \Longleftrightarrow \quad \forall \beta \in \Delta \exists \alpha \in \Gamma(\alpha \geqslant \beta)
\end{array}
$$

For readability we drop the subscript $H$ when attaching labels to rewrite steps as in $\uplus_{\vee}$. 
Example 47 Let $\geqslant$ denote the natural order on $\mathbb{N}$. Then $\{1\} \geqslant_{H}\{0,1\}$ and $\{1\} \geqslant_{H}$ $\{1,1,1\}=\{1\}$ but $\{5,4\} \ngtr_{H}\{5,3\}$.

The following lemma states obvious properties of Hoare preorders which we implicitly use in the sequel.

\section{Lemma 48 Let $\left(\geqslant_{H},>_{H}\right)$ be a Hoare preorder.}

1. If $(\geqslant,>)$ is a monotone reduction pair then $\left(\geqslant_{H},>_{H}\right)$ is a monotone reduction pair.

2. If $\Gamma \supseteq \Gamma^{\prime}$ then $\Gamma \geqslant{ }_{H} \Gamma^{\prime}$.

3. If $\Gamma>_{H} \Gamma^{\prime}$ and $\Delta>_{H} \Delta^{\prime}$ then $\Gamma \cup \Delta>_{H} \Gamma^{\prime} \cup \Delta^{\prime}$.

4. If $\Gamma \geqslant_{H} \Gamma^{\prime}$ and $\Delta \geqslant_{H} \Delta^{\prime}$ then $\Gamma \cup \Delta \geqslant_{H} \Gamma^{\prime} \cup \Delta^{\prime}$.

As we have seen in Section 3.2, constructing LL-labelings is quite a bit harder than constructing L-labelings, because of the duplicated steps in the (variable-left-linear) case (Fig. 2c). Here, we use weak LL-labelings for labeling single and parallel rewrite steps. Throughout this section we assume a given left-linear TRS $\mathcal{R}$, and a weak LL-labeling $\ell$ with corresponding labeling function for parallel steps $\ell^{\|}$, as introduced in the following definition.

Definition 49 We lift a weak LL-labeling $\ell$ to parallel steps $t \rightarrow^{P} \quad t^{\prime}$ as follows. For each $\pi \in P$, we have a rewrite step $t \rightarrow^{\pi} t^{\pi}$. We label $t \rightarrow^{P} t^{\prime}$ by $\ell^{\|}\left(t \rightarrow^{P} t^{\prime}\right)=$ $\left\{\ell\left(t \rightarrow^{\pi} t^{\pi}\right) \mid \pi \in P\right\}$.

So a parallel rewrite step is labeled by the set of the labels of the single steps making up the parallel step. We indicate labels along with the step, writing $t \rightarrow_{\Gamma}^{P} t^{\prime}$.

The next example shows that the labels change when decomposing a parallel step into a sequence of single steps, i.e., the label of the parallel step may be different from the union of labels of the single steps. However, the proof of Lemma 51 reveals that for weak LL-labelings the labels never increase when sequencing a parallel step.

Example 50 Consider the rule $\mathrm{a} \rightarrow \mathrm{b}$ and the extension of the source labeling $\ell(s \rightarrow t)=s$

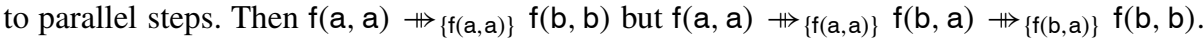
Clearly $\{f(a, a)\} \neq\{f(a, a), f(b, a)\}$. This effect is intrinsic to labelings that take the context of the rewrite step into account. On the other hand, the rule labeling gives $f(a, a) \nrightarrow\{1\}$ $f(b, b)$ and $f(a, a) \rightarrow_{\{1\}} f(b, a) \rightarrow_{\{1\}} f(b, b)$ with $\{1\}=\{1,1\}$, because the labels are independent of the context.

The following lemma is the key to show that even for parallel rewriting overlaps due to Fig. 2a (parallel) and Fig. 2c (variable-left-linear) are decreasing.

Lemma 51 1. Let $t_{1}{ }_{\Gamma}^{P} \nleftarrow s \leftrightarrow{ }_{\Delta}^{Q} t_{2}$ with $P \| Q$. There is a term $u$ such that $s \leftrightarrow{ }_{\Gamma \cup \Delta}^{P \cup Q} u$ and $t_{1} \ggg{ }_{\Delta^{\prime}}^{Q} u \Gamma_{\Gamma^{\prime}}^{P} \ll t_{2}$, where $\Gamma \geqslant_{H} \Gamma^{\prime}$ and $\Delta \geqslant_{H} \Delta^{\prime}$.

2. Let $s \boxplus s^{\prime}$ and $\sigma(x) \boxplus \sigma^{\prime}(x)$ for all $x \in \mathcal{V}$, so that there are parallel rewrite steps $s \sigma^{\prime}{ }_{\Gamma}^{P} \# s \sigma \boxplus \underset{\Delta}{Q} s^{\prime} \sigma$. Then $s \sigma^{\prime} \prod_{\Delta^{\prime}}^{Q} s^{\prime} \sigma^{\prime} \Gamma^{\prime} \# s^{\prime} \sigma$ and $\Gamma \geqslant_{H} \Gamma^{\prime}, \Delta \geqslant_{H} \Delta^{\prime}$. Furthermore, if $\sigma(x)=\sigma^{\prime}(x)$ for all $x \in \mathcal{V} \operatorname{ar}\left(\left.s^{\prime}\right|_{Q}\right)$ then $s \sigma \prod_{\Sigma} s^{\prime} \sigma^{\prime}$ for some $\Sigma \subseteq \Gamma \cup \Delta$. 
Proof 1 . First note that since $P \| Q$, a term $u$ with $s \oiint^{P \cup Q} u$ exists. We have

$$
\begin{aligned}
\ell^{\|}(s \stackrel{P \cup Q}{\longrightarrow} u) & =\left\{\ell\left(s \stackrel{\pi}{\rightarrow} s^{\pi}\right) \mid \pi \in P \cup Q\right\} \\
& =\left\{\ell\left(s \stackrel{\pi}{\rightarrow} s^{\pi}\right) \mid \pi \in P\right\} \cup\left\{\ell\left(s \stackrel{\pi}{\rightarrow} s^{\pi}\right) \mid \pi \in Q\right\} \\
& =\ell^{\|}\left(s \stackrel{P}{\rightarrow} t_{1}\right) \cup \ell^{\|}\left(s \stackrel{Q}{\rightarrow} t_{2}\right)=\Gamma \cup \Delta
\end{aligned}
$$

by definition. To establish $t_{1} \leftrightarrow \underset{\Delta^{\prime}}{Q} u \stackrel{P}{\Gamma^{\prime}}$ 世 $t_{2}$, we use induction on $|P|+|Q|$. We consider several base cases. If $|P|=0$ or $|Q|=0$ then the result follows by definition of parallel rewriting. If $|P|=|Q|=1$ the result follows from the fact that $\ell$ is a weak LL-labeling, Definition 10(1) (Fig. 2a). For the induction step, assume without loss of generality that $|P|>1$ and let $P=\{\pi\} \uplus P^{\prime}$. The proof is illustrated in Fig. 4. The parallel $P$-step can be decomposed into a $\pi$-step and a $P^{\prime}$-step. Since $\{\pi\}, P^{\prime} \subseteq P$, the labels are less than or equal to $\Gamma$. Then we apply the induction hypothesis to the peaks

i $\quad s^{P^{\prime}} \underset{\mathrm{V} / \Gamma}{P^{\prime}} \ll s \uplus_{\mathrm{V} / \Gamma}^{\{\pi\}} s^{\pi}$ yielding $s^{\pi} \uplus_{\mathrm{V} / \Gamma}^{P^{\prime}} t_{1}$,

ii $s^{\pi} \underset{V / \Gamma}{\{\pi\}} \ll s \gg{ }_{\Delta}^{Q} t_{2}$ yielding $t_{2} \gg \mathrm{V} / \Gamma^{\{\pi\}} t_{2}^{\pi}$ and $s^{\pi} \mapsto_{\mathrm{V} / \Delta}^{Q} t_{2}^{\pi}$,

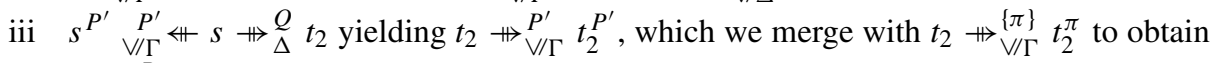
$t_{2} \mapsto \underset{\mathrm{V} / \Gamma}{P} u$, noting that the union of two sets from $\mathrm{V} / \Gamma$ is again in $\mathrm{V} / \Gamma$, and finally

iv $t_{1} \underset{\vee / \Gamma}{P^{\prime}} \ll s^{\pi} \mapsto \underset{\mathrm{V} / \Delta}{Q} t_{2}^{\pi}$ yielding $t_{1} \mapsto \underset{\mathrm{V} / \Delta}{Q} u$.

2. The existence of parallel rewrite steps $s \sigma^{\prime} \gg s^{\prime} \sigma^{\prime}$ and $s^{\prime} \sigma \boxplus s^{\prime} \sigma^{\prime}$ follows easily from the definition of parallel steps. We establish $\Gamma \geqslant_{H} \Gamma^{\prime}$ and $\Delta \geqslant_{H} \Delta^{\prime}$ by induction on $|Q|$. The reasoning for the induction step $(|Q|>1)$ is very similar to the induction step in item 1, cf. Figure 5a: Taking $Q=\{\pi\} \uplus Q^{\prime}$, we split $s \sigma \rightarrow_{\Delta}^{Q} s^{\prime} \sigma$ into $s \sigma \mapsto \frac{\{\pi\}}{\mathrm{V} / \Delta} s^{\pi} \sigma$ and $s \sigma \mapsto \underset{\mathrm{V} / \Delta}{Q^{\prime}} s^{Q^{\prime}} \sigma$. We apply the induction hypothesis to the peaks

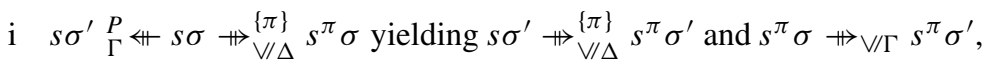

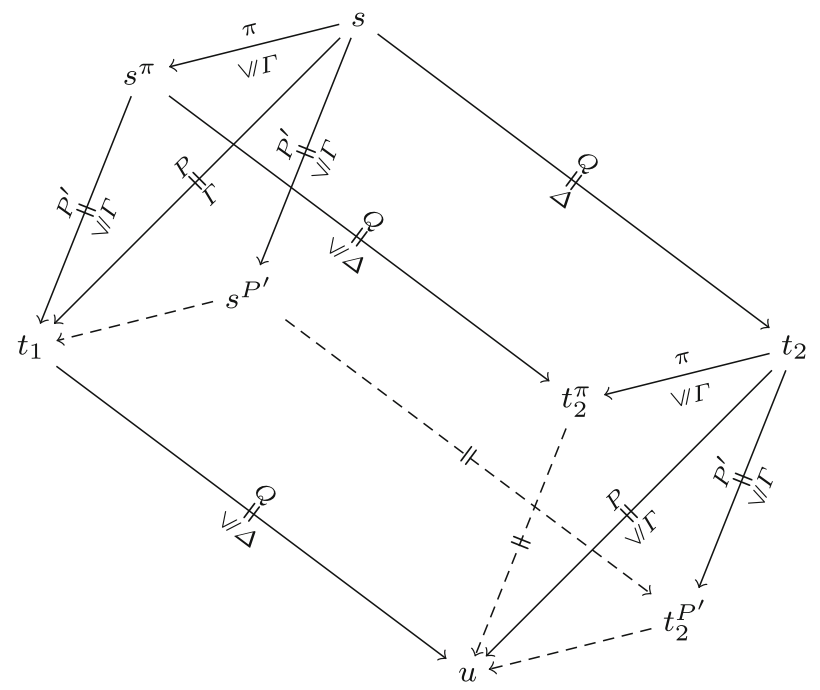

Fig. 4 Weak LL-labeling applied to parallel steps 


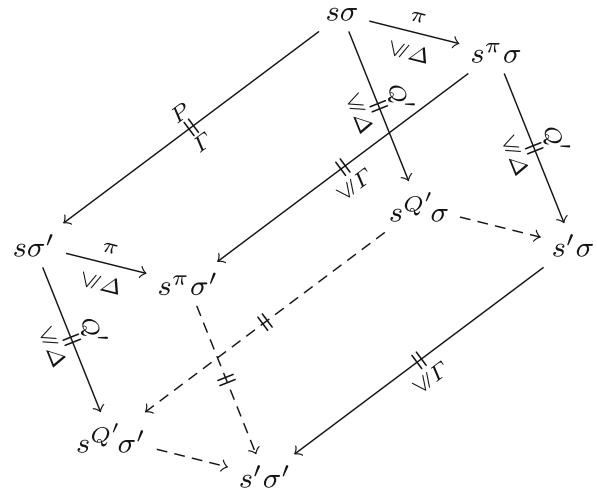

(a) split base step

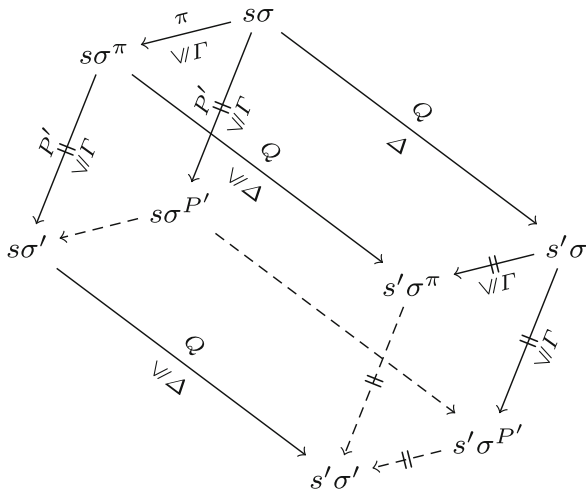

(b) split substitution

Fig. 5 Weak LL-labeling applied to nested parallel steps

ii $s \sigma^{\prime} \underset{\Gamma}{P} \# s \sigma \boxplus \underset{\mathrm{V} / \Delta}{Q^{\prime}} s^{Q^{\prime}} \sigma$ yielding $s \sigma^{\prime} \rightarrow \underset{\mathrm{V} / \Delta}{Q^{\prime}}{ }_{s} Q_{\sigma^{\prime}}^{\prime}$, which can be merged with $s \sigma^{\prime} \ggg \frac{\{\pi\}}{\vee / \Delta} s^{\pi} \sigma^{\prime}$ to obtain $s \sigma^{\prime} \ggg \underset{\mathrm{V} / \Delta}{Q} s^{\prime} \sigma^{\prime}$, and finally

iii $s^{\pi} \sigma^{\prime} \mathrm{V} / \Gamma+s^{\pi} \sigma \prod_{\mathrm{V} / \Delta}^{Q^{\prime}} s^{\prime} \sigma$ yielding $s^{\prime} \sigma \boxplus \mathrm{V} / \Gamma s^{\prime} \sigma^{\prime}$, where $s^{\pi} \sigma \prod_{\mathrm{V} / \Delta}^{Q^{\prime}} s \sigma^{\prime}$ is obtained from part 1 of this lemma applied to $s^{Q^{\prime}} \underset{V / \Delta}{Q^{\prime}} \ll s \sigma \mapsto_{\mathrm{V} / \Delta}^{\{\pi\}} s^{\pi} \sigma$.

This concludes the induction step. If $|Q|=0$, there is nothing to show, so only the base case $|Q|=1$ remains. Note that because $\mathcal{R}$ is left-linear, we may assume without loss of generality that $s$ is linear. Therefore, every rewrite step of $s \sigma \pitchfork^{P} s \sigma^{\prime}$ can be performed by modifying $\sigma$. For $P^{\prime} \subseteq P$, we write $\sigma^{P^{\prime}}$ for the substitution $\tau$ that satisfies $s \sigma \gg{ }^{P^{\prime}} s \tau$, and proceed by induction on $|P|$. For the induction step $(|P|>1)$, the argument is again almost the same as before, cf. Figure 5b. Let $P=\{\pi\} \uplus P^{\prime}$. We split $s \sigma \rightarrow_{\Gamma}^{P} s \sigma^{\prime}$ into $s \sigma \boxplus \frac{\{\pi\}}{V / \Gamma} s \sigma^{\pi}$ and $s \sigma \boxplus \frac{V^{\prime} / \Gamma}{P^{\prime}} s \sigma^{P^{\prime}}$. Next we apply the induction hypothesis to the peaks

i $\quad s \sigma^{\pi} \underset{\vee / \Gamma}{\{\pi} \ll s \sigma \rightarrow \underset{\Delta}{Q} s^{\prime} \sigma$ yielding $s \sigma^{\pi} \rightarrow \underset{\mathrm{V} / \Delta}{Q} s^{\prime} \sigma^{\pi}$ and $s^{\prime} \sigma \prod_{\mathrm{V} / \Gamma} s^{\prime} \sigma^{\pi}$,

ii $s \sigma^{P^{\prime}} \underset{V / \Gamma}{P^{\prime}} \ll s \sigma \rightarrow{ }_{\Delta}^{Q} s^{\prime} \sigma$ yielding $s^{\prime} \sigma \quad \uplus_{\mathrm{V} / \Gamma} s^{\prime} \sigma^{P^{\prime}}$, which can be merged with $s^{\prime} \sigma \ggg \mathrm{V} / \Gamma s^{\prime} \sigma^{\pi}$ to obtain $s^{\prime} \sigma \prod_{\mathrm{V} / \Gamma} s^{\prime} \sigma^{\prime}$, and finally

iii $s \sigma^{\prime} \underset{\mathrm{V} / \Gamma}{P^{\prime}} \ll+s \sigma^{\pi} \rightarrow \underset{\mathrm{V} / \Delta}{Q} s^{\prime} \sigma^{\pi}$ yielding $s \sigma^{\prime} \rightarrow \underset{\mathrm{V} / \Delta}{Q} s^{\prime} \sigma^{\prime}$, where $s \sigma^{\pi} \rightarrow_{\mathrm{V} / \Gamma}^{P^{\prime}} s \sigma^{\prime}$ is obtained

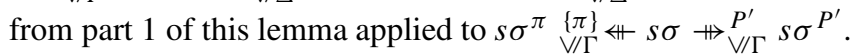

This concludes the induction step. If $|P|=0$ then there is nothing to show. Finally, if $|P|=|Q|=1$, then we are left with a parallel or variable overlap, and we conclude by Definition 10(1) or 10(2), respectively. This concludes the proof that $\Gamma \geqslant_{H} \Gamma^{\prime}$ and $\Delta \geqslant_{H} \Delta^{\prime}$. Now if $\sigma(x)=\sigma^{\prime}(x)$ for all $x \in \mathcal{V} \operatorname{ar}\left(\left.s^{\prime}\right|_{Q}\right)$, then $s^{\prime} \sigma \boxplus P^{\prime} s^{\prime} \sigma^{\prime}$ satisfies $P^{\prime} \| Q$. Performing the same rewrite steps on $s \sigma$, we obtain a parallel rewrite step $s \sigma \leftrightarrow P^{\prime} s^{\prime \prime}$ with $P^{\prime} \subseteq P$ and therefore $\Gamma^{\prime \prime}=\ell^{\|}\left(s \sigma \boxplus^{P^{\prime}} s^{\prime \prime}\right) \subseteq \ell^{\|}\left(s \sigma \pitchfork^{P} s \sigma^{\prime}\right)=\Gamma$. Finally, using the first part of this lemma, we can combine the two parallel steps from $s \sigma$ into a single one, $s \sigma \mapsto_{\Gamma^{\prime \prime} \cup \Delta}^{P^{\prime} \cup Q} s^{\prime} \sigma^{\prime}$ with $\Sigma=\Gamma^{\prime \prime} \cup \Delta \subseteq \Gamma \cup \Delta$ as claimed.

Only Definition 10(1) was used in the proof of Lemma 51(1). This fact can be exploited for an alternative characterization of weak LL-labelings. 


\section{Corollary 52 Let $\ell$ be a labeling. Then $\ell$ is a weak LL-labeling if and only if}

1. in Fig. $2 a, \alpha \geqslant \gamma$ and $\beta \geqslant \delta$, and

2. in Fig. $2 c, \beta \geqslant \delta$ and $\{\alpha\} \geqslant_{H} \ell^{\|}(u \ggg v)$.

Proof Assume that $\ell$ is a weak LL-labeling. The first condition of this lemma is identical to Definition 10(1). For the second condition, $\beta \geqslant \delta$ follows from Definition 10(2). To establish $\{\alpha\} \geqslant_{H} \ell^{\|}\left(u \rightarrow^{P} v\right)$, we need to show that $\alpha \geqslant \ell\left(u \rightarrow^{\pi} u^{\pi}\right)$ for all $\pi \in P$. For each $\pi$, we can arrange that $\ell\left(u \boxplus^{\pi} u^{\pi}\right)=\gamma_{1}$ by choosing $u \boxplus^{\pi} u^{\pi}$ as the first step in the permutation of $u \nrightarrow v$, and then $\alpha \geqslant \gamma_{1}$ follows from Definition 10(2), establishing the claim.

Next assume that $\ell$ satisfies the conditions of this lemma. Then the condition of Definition 10(1) holds. To show the conditions of Definition 10(2), note that $\beta \geqslant \delta$ holds by assumption. Consider the parallel rewrite step $u \rightarrow^{P} v$ and a permutation $\pi_{1}, \ldots, \pi_{n}$ of $P$. We can decompose $u \rightarrow^{P} v$ into $u=u_{0} \rightarrow_{\gamma_{1}}^{\pi_{1}} u_{1} \rightarrow \gamma_{2}^{\pi_{2}} \cdots \rightarrow_{\gamma_{n}}^{\pi_{n}} u_{n}=v$. By Lemma 51(1) applied to the peaks

$$
\cdot \underset{\mathrm{V} /\{\alpha\}}{\stackrel{\left\{\pi_{i}\right\}}{\|}} u \stackrel{\left\{\pi_{1}, \ldots, \pi_{i-1}\right\}}{\mathrm{V} /\{\alpha\}} u_{i-1}
$$

we obtain $u_{i-1} \rightarrow \mathbb{V}_{\mathrm{V} /\{\alpha\}}^{\left\{\pi_{i}\right\}} u_{i}$, i.e., $\{\alpha\} \geqslant \geqslant_{H}\left\{\gamma_{i}\right\}$, which is equivalent to $\alpha \geqslant \gamma_{i}$. Hence we conclude $\alpha \geqslant \bar{\gamma}$.

The following lemma is used to reduce the number of parallel peaks that have to be considered in the proof of Theorem 56.

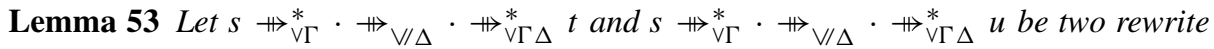
sequences such that all rewrite steps in the sequence to $t$ are at or below a position $p$ and the rewrite steps in the sequence to $u$ are parallel to $p$. Then the two rewrite sequences can

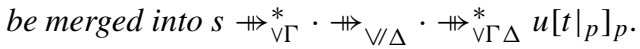

Proof Let the two sequences be $s \rightarrow_{\vee \Gamma}^{*} t_{1} \rightarrow_{\vee / \Delta} t_{2} \rightarrow_{\vee \Gamma \Delta}^{*} t$ and $s \rightarrow_{\vee \Gamma}^{*} u_{1} \rightarrow_{V / \Delta}$ $u_{2} \mapsto_{\vee \Gamma \Delta}^{*} u$. Using Lemma 51(1) repeatedly, we can derive a sequence

$$
s \underset{\mathrm{V} \Gamma}{\stackrel{*}{\leftrightarrow}} u_{1}\left[\left.t_{1}\right|_{p}\right]_{p} \underset{\mathrm{V} / \Delta}{\longrightarrow} u_{2}\left[\left.t_{2}\right|_{p}\right]_{p} \underset{\mathrm{V} \Gamma \Delta}{\stackrel{*}{\leftrightarrow}} u\left[\left.t\right|_{p}\right]_{p}
$$

which establishes the claim.

In order to perform a critical pair analysis for parallel rewrite steps, we need parallel critical pairs $[9,26]$.

Definition 54 Let $l \rightarrow r$ be a rule in a TRS $\mathcal{R}$ and $P$ be a non-empty set of pairwise parallel redex patterns such that every $\pi \in P$ critically overlaps with $l$. By choosing variants of rules from $\mathcal{R}$ appropriately, we may assume that the sets $\mathcal{V} \operatorname{ar}\left(l_{\pi}\right)$ for $\pi \in P$ and $\mathcal{V} \operatorname{ar}(l)$ are pairwise disjoint. Assume that the unification problem $\left\{\left.l\right|_{p_{\pi}} \approx l_{\pi} \mid \pi \in P\right\}$ has a solution and let $\sigma$ be a most general unifier. Then there is a unique term $l_{P}$ such that $l \sigma \pitchfork^{P} l_{P}$. We call $l_{P} \nleftarrow \rtimes \rightarrow r \sigma$ a parallel critical pair, and $l_{P} \nleftarrow l \sigma \rightarrow r \sigma$ a parallel critical peak.

Note that every standard critical pair also is a parallel critical pair. The following lemma states how critical pair analysis for a peak consisting of a parallel and a root rewrite step is done. It is a straightforward extension of [9, Lemma 4.7]. 
Lemma 55 Let $\mathcal{R}$ be a left-linear TRS and $t^{P} \nleftarrow s \rightarrow^{\pi} u$ with $p_{\pi}=\epsilon$. Then either $P \perp \pi$ or there are substitutions $\sigma \boxplus \sigma^{\prime}$ and a parallel critical pair $t^{\prime} \nleftarrow \rtimes \rightarrow u^{\prime}$ such that $t=t^{\prime} \sigma^{\prime} P \backslash P^{\prime} \ll t^{\prime} \sigma P^{\prime} \nLeftarrow s \rightarrow u^{\prime} \sigma=u$ with $P^{\prime} \subseteq P$.

Note that left-linearity is essential for the substitutions $\sigma$ and $\sigma^{\prime}$ to exist in Lemma 55. We are now ready to state and prove the main theorem of this section.

Theorem 56 A left-linear TRS $\mathcal{R}$ is confluent if all its parallel critical peaks $t{ }_{\Gamma}^{P} \pi s \rightarrow_{\Delta} u$ can be joined decreasingly as

$$
t \underset{\mathrm{V} \Gamma}{\stackrel{*}{\longrightarrow}} \cdot \underset{\mathrm{V} / \Delta}{\mathrm{H}} \cdot \frac{*}{\mathrm{~V} \Gamma \Delta} \cdot \underset{\mathrm{V} \Gamma \Delta}{\stackrel{*}{\longrightarrow}} v \underset{\mathrm{V} / \Gamma}{\stackrel{Q}{\Perp}} \cdot \underset{\mathrm{V} \Delta}{\stackrel{*}{\leftarrow}} u
$$

such that $\mathcal{V} \operatorname{ar}\left(\left.v\right|_{Q}\right) \subseteq \mathcal{V} \operatorname{ar}\left(\left.s\right|_{P}\right)$.

Proof We show that $\pitchfork$ is decreasing, which implies confluence of $\mathcal{R}$. Let $t{ }_{\Gamma}^{P} \nleftarrow s \boxplus \frac{Q}{\Delta} u$. It suffices to show that

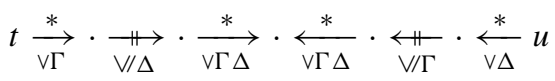

Below we show that (2) holds whenever $P=\{\pi\}$ or $Q=\{\pi\}$ with $p_{\pi}=\epsilon$. Then for all

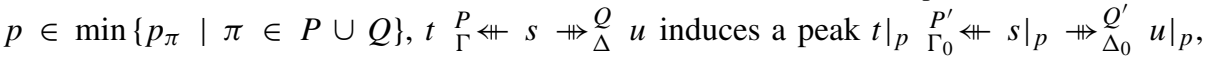
where $P^{\prime}=\{\pi\}$ or $Q^{\prime}=\{\pi\}$ for some $\pi$ with $p_{\pi}=\epsilon$. So for each $p$, we obtain a joining sequence for $\left.t\right|_{p}$ and $\left.u\right|_{p}$ of shape (2). By the monotonicity of labelings, this results in joining sequences

$$
s\left[\left.t\right|_{p}\right]_{p} \underset{\mathrm{V} \Gamma}{\stackrel{*}{\longrightarrow}} \cdot \underset{\mathrm{V} / \Delta}{\longrightarrow} \cdot \frac{*}{\mathrm{~V} \Gamma \Delta} \cdot \underset{\mathrm{V} \Gamma \Delta}{\stackrel{*}{\longrightarrow}} \cdot \underset{\mathrm{V} / \Gamma}{\overleftrightarrow{\mathrm{V} \Delta}} s\left[\left.u\right|_{p}\right]_{p}
$$

which are mutually parallel since the positions $p \in \min (P \cup Q)$ are mutually parallel. By repeated application of Lemma 53 those sequences can be combined into a single sequence of the same shape.

In order to show (2) for $P=\{\pi\}$ or $Q=\{\pi\}$ with $p_{\pi}=\epsilon$, assume without loss of generality that $Q=\{\pi\}$. If $P \perp \pi$ then $s=l_{\pi} \sigma$ and, because $l_{\pi}$ is linear, there is a substitution $\sigma^{\prime}$ with $t=l_{\pi} \sigma^{\prime}$ and $\sigma(x) \boxplus \sigma^{\prime}(x)$ for all variables $x \in \mathcal{V}$. We conclude by Lemma 51(2). Otherwise $P$ and $\pi$ overlap, and by Lemma 55, there are a parallel critical

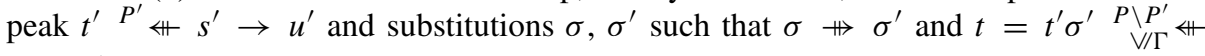
$t^{\prime} \sigma \underset{V / \Gamma}{P^{\prime}} \ll s^{\prime} \sigma=s \rightarrow{ }_{\Delta}^{\epsilon} u^{\prime} \sigma=u$ with $P^{\prime} \subseteq P$. This case is illustrated in Fig. 6. By assumption there are $u^{\prime \prime}, v$ and $v^{\prime}$ with $\mathcal{V} \operatorname{ar}\left(\left.v\right|_{Q^{\prime}}\right) \subseteq \mathcal{V} \operatorname{ar}\left(\left.s\right|_{P^{\prime}}\right)$ such that we can join $t^{\prime}$ and $u^{\prime}$ decreasingly, and consequently, using the stability of labelings we obtain

$$
t^{\prime} \sigma \underset{\mathrm{V} \Gamma}{\stackrel{*}{\longrightarrow}} \cdot \underset{\mathrm{V} / \Delta}{\Perp} \cdot \underset{\mathrm{V} \Gamma \Delta}{*} v^{\prime} \sigma \underset{\mathrm{V} \Gamma \Delta}{\stackrel{*}{\leftrightarrows}} v \sigma \underset{\mathrm{V} / \Gamma}{\stackrel{Q^{\prime}}{\dddot{H}}} u^{\prime \prime} \sigma \underset{\mathrm{V} \Delta}{\stackrel{*}{*}} u^{\prime} \sigma=u
$$

Furthermore, making repeated use of Lemma 51(2),

$$
t=t^{\prime} \sigma^{\prime} \underset{\mathrm{V} \Gamma}{\stackrel{*}{\longrightarrow}} \cdot \underset{\mathrm{V} / \Delta}{\longrightarrow} \cdot \underset{\mathrm{V} \Gamma \Delta}{\stackrel{*}{\longrightarrow}} v^{\prime} \sigma^{\prime} \underset{\mathrm{V} \Gamma \Delta}{\stackrel{*}{4}} v \sigma^{\prime} \underset{\mathrm{V} / \Gamma}{\stackrel{\pi}{L}} v \sigma
$$

Notably, the step $v \sigma \boxplus_{\mathrm{V} / \Gamma} v \sigma^{\prime}$ is obtained from $s^{\prime} \sigma \oiint_{\mathrm{V} / \Gamma} s^{\prime} \sigma^{\prime}$ by passing through the rewrite sequence $s^{\prime} \sigma \rightarrow u^{\prime} \sigma \rightarrow^{*} u^{\prime \prime} \sigma \boxplus v \sigma$. We have $\sigma(x)=\sigma^{\prime}(x)$ for $x \in \mathcal{V} \operatorname{ar}\left(\left.s\right|_{P^{\prime}}\right)$ for 


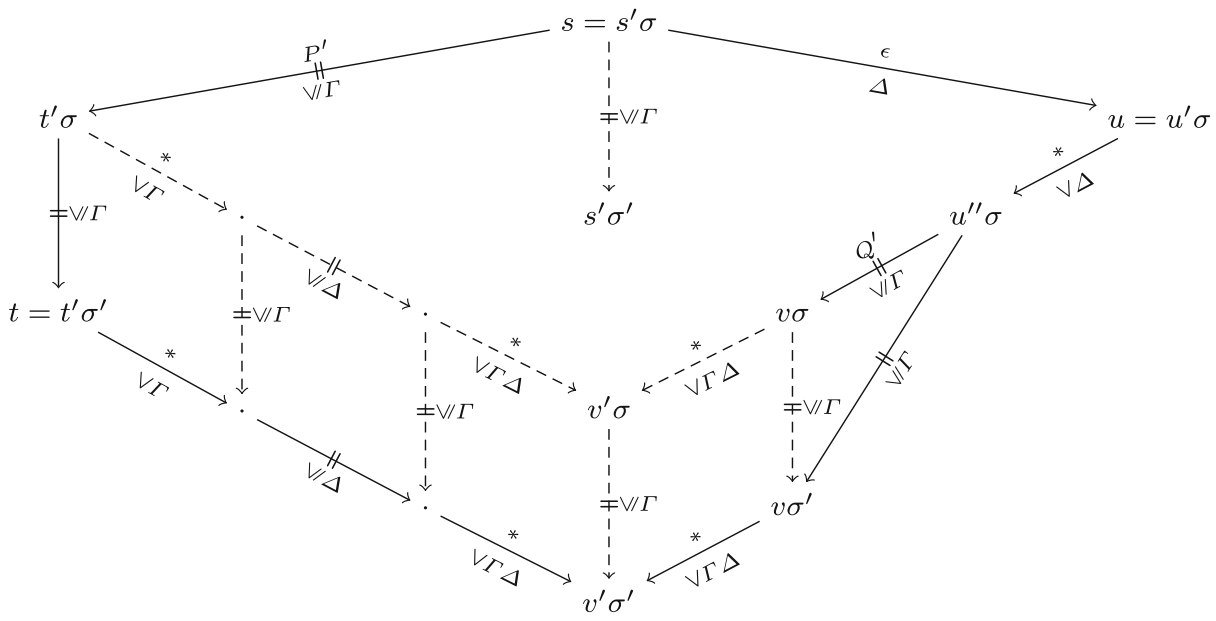

Fig. 6 Part of the proof of Theorem 56

otherwise $s \rightarrow_{\Gamma} t$ would not be a parallel step. Together with $\mathcal{V} \operatorname{ar}\left(\left.v\right|_{Q^{\prime}}\right) \subseteq \mathcal{V} \operatorname{ar}\left(\left.s\right|_{P^{\prime}}\right)$, the parallel steps $u^{\prime \prime} \sigma \oiint_{\mathrm{V} / \Gamma} v \sigma$ and $v \sigma \oiint_{\mathrm{V} / \Gamma} v \sigma^{\prime}$ can be combined into a single $\boxplus_{\mathrm{V} / \Gamma}$ step by Lemma 51(2). Thus we can join $t$ and $u$ decreasingly with common reduct $v^{\prime} \sigma^{\prime}$, completing the proof.

To conclude the section we demonstrate Theorem 56 on two examples. Both are based on rule labeling.

Example 57 Consider the TRS $\mathcal{R}$ consisting of the following five rules with labels $2>1>0$ :

$$
\mathrm{a} \underset{1}{\rightarrow} \mathrm{b} \quad \mathrm{b} \underset{0}{\rightarrow} \mathrm{a} \quad \mathrm{f}(\mathrm{a}, \mathrm{a}) \underset{1}{\rightarrow} \mathrm{c} \quad \mathrm{f}(\mathrm{b}, \mathrm{b}) \underset{2}{\underset{\mathrm{c}}{\mathrm{c}}} \quad \mathrm{h}(x) \underset{0}{\rightarrow} \mathrm{h}(\mathrm{f}(x, x))
$$

There are six parallel critical peaks that can all be joined decreasingly as required by Theorem 56:

$$
\begin{aligned}
& f(b, a) \underset{\{1\}}{\stackrel{t}{t}} f(a, a) \underset{\{1\}}{\longrightarrow} c: \quad f(b, a) \underset{\{0\}}{\longrightarrow} f(a, a) \underset{\{1\}}{\longrightarrow} c \\
& f(a, b) \underset{\{1\}}{\stackrel{4}{t}} f(a, a) \underset{\{1\}}{\longrightarrow} c: \quad f(a, b) \underset{\{0\}}{\longrightarrow} f(a, a) \underset{\{1\}}{\longrightarrow} c \\
& f(b, b) \underset{\{1\}}{\overleftrightarrow{4}} f(a, a) \underset{\{1\}}{\longrightarrow} c: \quad f(b, b) \underset{\{0\}}{\longrightarrow} f(a, a) \underset{\{1\}}{\longrightarrow} c \\
& f(a, b) \underset{\{0\}}{\stackrel{\#}{f}} f(b, b) \underset{\{2\}}{\longrightarrow} c: \quad f(a, b) \underset{\{0\}}{\longrightarrow} f(a, a) \underset{\{1\}}{\longrightarrow} c \\
& f(b, a) \underset{\{0\}}{\stackrel{\pi}{f}} f(b, b) \underset{\{2\}}{\longrightarrow} c: \quad f(b, a) \underset{\{0\}}{\longrightarrow} f(a, a) \underset{\{1\}}{\longrightarrow} c
\end{aligned}
$$

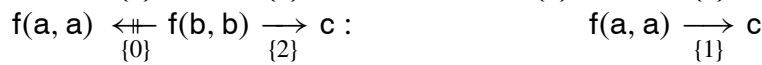

Therefore, $\mathcal{R}$ is confluent. 
Example 58 Let $\mathcal{R}$ be the TRS (Cops \#62) consisting of the (labeled) rules

$$
\begin{aligned}
& x-0 \rightarrow 0 \\
& 0<\mathrm{s}(x) \underset{0}{\rightarrow} \text { true } \\
& \operatorname{gcd}(x, 0) \rightarrow \overrightarrow{0}^{x} \\
& \text { if(true, } x, y) \underset{0}{\rightarrow} \quad x \\
& \bmod (x, 0) \rightarrow \overrightarrow{0}^{x} \\
& 0-x \rightarrow 0 \\
& x<0 \underset{0}{\rightarrow} \text { false } \mathrm{s}(x)<\mathrm{s}(y) \underset{0}{\overrightarrow{0}} x<y \\
& \operatorname{gcd}(0, x) \rightarrow{ }_{0} x \\
& \text { if(false, } x, y) \rightarrow \overrightarrow{0} \quad y \\
& \bmod (0, x) \underset{0}{0} 0 \\
& \bmod (x, \mathbf{s}(y)) \overrightarrow{1} \text { if }(x<\mathrm{s}(y), x, \bmod (x-\mathbf{s}(y), \mathbf{s}(y)))
\end{aligned}
$$

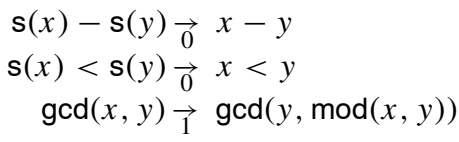

There are 12 critical pairs, 6 of which are trivial. One easily verifies that the remaining 6 pairs can be joined decreasingly, using the order $1>0$. Hence the confluence of $\mathcal{R}$ follows from Theorem 56. Even though $\mathcal{R}$ lacks proper parallel critical pairs, none of the other results in this paper applies. Note that the preconditions for Corollaries 16, 25, and 31 are not satisfied as $\mathcal{R}_{d} / \mathcal{R}_{n d}, \star(\mathcal{R})$, and $\mathcal{R}^{\triangle}$ are non-terminating (due to the rules with label 1). Finally, persistence cannot rule out variable overlaps (of the duplicating mod rule below the variable $x$ ) and hence Theorems 41 and 43 based on the rule labeling fail.

\section{Assessment}

In this section we relate the results from this article to each other (Section 5.1) and to the recent literature [1, 11] (Section 5.2).

\subsection{Interrelationships}

The main results for left-linear systems presented in this article can be divided into three classes. Those that require relative termination as a precondition (Corollaries 16, 25, and 31), those exploiting persistence (Theorems 41 and 43), and those considering parallel rewriting (Theorem 56). Figure 7a demonstrates that these three classes are incomparable. The same holds when focusing on the results relying on relative termination, cf. Fig. $7 \mathrm{~b}$. Note that the regions where only one class is applicable can be populated with examples

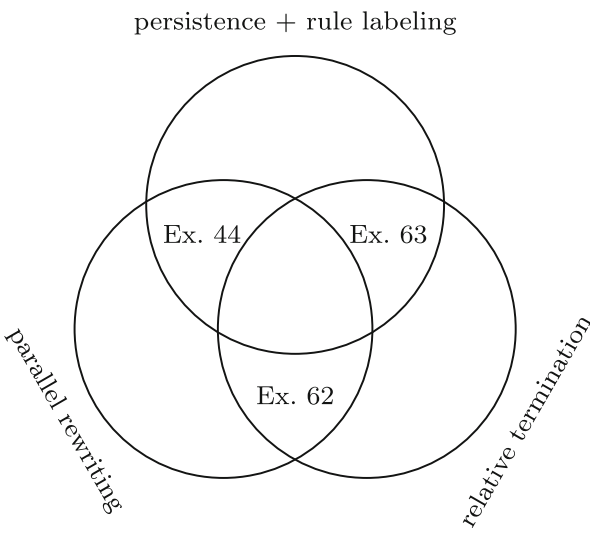

(a) All

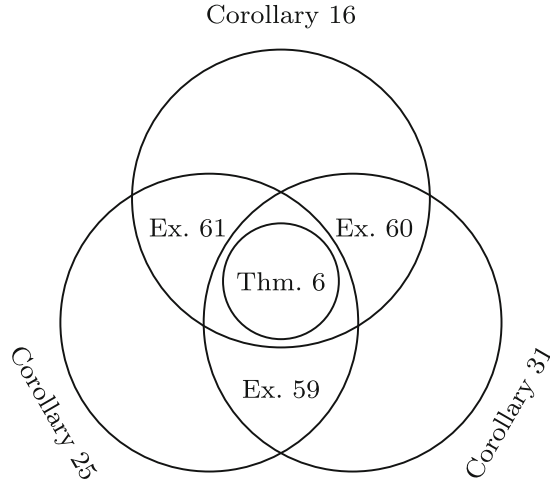

(b) Relative termination

Fig. 7 Interrelationships 
using Toyama's celebrated modularity result [25], e.g., the disjoint union (after renaming function symbols) of the TRSs in Examples 62 and 63 can only be handled by the approach based on relative termination. We discuss the interrelationships in more detail below.

First we observe that Corollaries 16, 25, and 31 subsume Theorem 6 since the preconditions of the corollaries evaporate for linear systems. The inclusion is strict since Theorem 6 cannot deal with the rule $\mathrm{f}(x) \rightarrow \mathrm{g}(x, x)$, while all the corollaries can. Furthermore, Theorem 6 is subsumed by Theorem 41, which, if restricted to weak LL-labelings, is subsumed by Theorem 43 .

The following three examples show that Corollaries 16, 25, and 31 are pairwise incomparable in power (for an overview see Fig. 7b).

Example 59 Consider the TRS $\mathcal{R}$ consisting of the following rules

$$
\begin{array}{rlrl}
\mathrm{f}(\mathrm{h}(x)) & \rightarrow \mathrm{k}(\mathrm{g}(\mathrm{f}(x), x, \mathrm{f}(\mathrm{h}(\mathrm{a})))) & \mathrm{f}(x) \rightarrow \mathrm{a} & \mathrm{a} \rightarrow \mathrm{b} \\
\mathrm{k}(x) \rightarrow \mathrm{c} & \mathrm{b} \rightarrow \perp & \mathrm{c} \rightarrow \perp
\end{array}
$$

This TRS has one critical peak (modulo symmetry). Since $\mathcal{R}_{d} / \mathcal{R}_{n d}$ is non-terminating, Corollary 16 does not apply. For Corollary 25 observe that $\star(\mathcal{R})$ is terminating using the interpretation $\mathrm{h}_{1 \mathbb{N}}(x)=x+1$ and the identify function for all other function symbols. To show decreasingness we use the labeling $\ell_{\star} \times \ell_{\mathrm{rl}}^{i}$ with $i(\mathrm{f}(x) \rightarrow \mathrm{a})=1$ and all other rules receive label 0. The critical peak $t=\mathrm{a}_{x, 1} \leftarrow \mathrm{f}(\mathrm{h}(x)) \rightarrow_{x, 0} \mathrm{k}(\mathrm{g}(\mathrm{f}(x), x, \mathrm{f}(\mathrm{h}(\mathrm{a}))))=u$ is closed decreasingly by $t \rightarrow_{x, 0} \mathrm{~b} \rightarrow_{x, 0} \perp x, 0 \leftarrow \mathrm{c}_{x, 0} \leftarrow u$. Corollary 31 also applies since the polynomial interpretation with $\mathrm{h}_{\mathbb{N}}(x)=3 x+1$ and interpreting all other function symbols by the sum of its arguments establishes termination of $\mathcal{R}^{\triangle} / \mathcal{R}$. When taking the identity for $\ell$ in Corollary 31 the critical peak $t=\mathrm{a}_{3 x+1} \leftarrow \mathrm{f}(\mathrm{h}(x)) \rightarrow 3 x+1 \mathrm{k}(\mathrm{g}(\mathrm{f}(x), x, \mathrm{f}(\mathrm{h}(\mathrm{a}))))=$ $u$ can be closed decreasingly by $t \rightarrow_{0} \mathrm{~b} \rightarrow_{0} \perp_{0} \leftarrow \mathrm{c}_{2 x+1} \leftarrow u$.

Example 60 It is easy to adapt the TRS from Example 18 such that $\star(\mathcal{R})$ becomes nonterminating. Consider the TRS $\mathcal{R}$

$$
1: \mathrm{b} \rightarrow \mathrm{a} \quad 2: \mathrm{a} \rightarrow \mathrm{b} \quad 3: \mathrm{f}(\mathrm{g}(x, \mathrm{a})) \rightarrow \mathrm{g}(\mathrm{f}(x), \mathrm{f}(\mathrm{g}(x, \mathrm{c})))
$$

for which termination of $\mathcal{R}_{d} / \mathcal{R}_{n d}$ is proved by LPO with precedence $\mathrm{f}>\mathrm{g}$ and $\mathrm{a} \sim \mathrm{b}>\mathrm{c}$. Corollary 16 applies since the rule labeling establishes decreasingness of the critical peak $t=\mathrm{f}(\mathrm{g}(x, \mathrm{~b}))_{2} \leftarrow \mathrm{f}(\mathrm{g}(x, \mathrm{a})) \rightarrow_{3} \mathrm{~g}(\mathrm{f}(x), \mathrm{f}(\mathrm{g}(x, \mathrm{c})))=u$ by the join $t \rightarrow_{1} \mathrm{f}(\mathrm{g}(x, \mathrm{a})) \rightarrow_{3} u$. Note that $\mathrm{f}_{1}\left(\mathrm{~g}_{1}(x)\right) \rightarrow \mathrm{g}_{2}\left(\mathrm{f}_{1}\left(\mathrm{~g}_{1}(x)\right)\right) \in \mathcal{R}_{>}^{\star}$ is non-terminating and hence Corollary 25 does not apply. ${ }^{3}$ For Corollary 31 the (above) termination proof establishes termination of $\mathcal{R}^{\Delta} / \mathcal{R}$ and $\ell_{\Delta}$ in combination with the rule labeling (taking rule numbers as labels) labels the

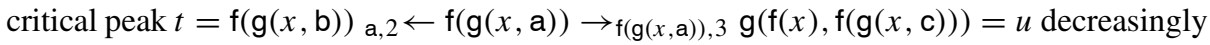
since $t \rightarrow_{\mathrm{b}, 1} \mathrm{f}(\mathrm{g}(x, \mathrm{a})) \rightarrow_{\mathrm{f}(\mathrm{g}(x, \mathrm{a}), 3} u$.

Example 61 Consider the TRS consisting of the rules

$$
\mathrm{a}(\mathrm{a}(\mathrm{c})) \rightarrow \mathrm{a}(\mathrm{b}(\mathrm{a}(\mathrm{c}))) \quad \mathrm{b}(x) \rightarrow \mathrm{h}(x, x)
$$

\footnotetext{
${ }^{3}$ We remark that it is easy to extend this example such that also $\star_{\star}^{\star}(\mathcal{R})$ is non-terminating; just consider the rule $\mathrm{f}(\mathrm{g}(x, \mathrm{a})) \rightarrow \mathrm{g}(\mathrm{f}(x), \mathrm{g}(\mathrm{f}(\mathrm{g}(x, \mathrm{c}), \mathrm{f}(\mathrm{g}(x, \mathrm{c})))))$.
} 
The TRS $\mathcal{R}$ has no critical peaks and is terminating by the following matrix interpretation over $\mathbb{N}^{2}$ :

$$
\begin{aligned}
& \mathrm{a}_{\mathbb{N}^{2}}(\vec{x})=\left(\begin{array}{ll}
1 & 1 \\
1 & 2
\end{array}\right) \vec{x}+\left(\begin{array}{l}
0 \\
3
\end{array}\right) \quad \mathrm{h}_{\mathbb{N}^{2}}(\vec{x}, \vec{y})=\left(\begin{array}{ll}
1 & 0 \\
0 & 0
\end{array}\right) \vec{x}+\left(\begin{array}{ll}
1 & 0 \\
0 & 0
\end{array}\right) \vec{y} \\
& \mathrm{~b}_{\mathbb{N}^{2}}(\vec{x})=\left(\begin{array}{ll}
2 & 0 \\
0 & 0
\end{array}\right) \vec{x}+\left(\begin{array}{l}
2 \\
0
\end{array}\right) \quad \mathrm{c}_{\mathbb{N}^{2}}=\left(\begin{array}{l}
0 \\
0
\end{array}\right)
\end{aligned}
$$

Hence also $\mathcal{R}_{d} / \mathcal{R}_{n d}$ is terminating, and by Corollary 16 the TRS $\mathcal{R}$ is confluent. Corollary 25 also applies since $\star(\mathcal{R})$ is terminating. The derivation $\mathrm{a}(\mathrm{a}(\mathrm{c})) \rightarrow$ $\mathrm{a}(\mathrm{b}(\mathrm{a}(\mathrm{c}))) \rightarrow_{\mathcal{R}^{\Delta}} \mathrm{a}(\mathrm{a}(\mathrm{c})) \rightarrow \cdots$ shows that $\mathcal{R}^{\Delta} / \mathcal{R}$ is non-terminating, so Corollary 31 does not apply.

Note that any simple monotone reduction pair showing termination of $\mathcal{R}_{d} / \mathcal{R}_{n d}$ will also establish termination of $\mathcal{R}^{\triangle} / \mathcal{R}$, because if $l \rightarrow x \in \mathcal{R}^{\triangle}$ then there is a rule $l \rightarrow r \in \mathcal{R}_{d}$ that duplicates $x$, whence $l>r \geqslant x$. Hence it is no surprise that Example 61 used a matrix interpretation of dimension 2.

Furthermore, the results on relative termination are incomparable with those on persistence and those based on parallel rewriting. To this end observe that the first rule of Example 44 violates all preconditions of Corollaries 16, 25, and 31 but Theorems 43 and 56 apply. Note that Theorem 43 based on arbitrary weak LL-labelings subsumes Corollaries 16 and 25 , since they produce LL-labelings which may be used to close problematic variable peaks decreasingly even without persistence. However, if restricted to the rule labeling the following TRS cannot be handled using persistence while each of the Corollaries 16, 25, and 31 as well as Theorem 56 succeeds.

Example 62 Consider the TRS consisting of the rules

$$
\text { 1: } \mathrm{f}(x, y, \mathrm{a}) \rightarrow \mathrm{f}(x, x, \mathrm{~b}) \quad 2: \mathrm{f}(\mathrm{f}(x, y, \mathrm{~b}), z, \mathrm{c}) \rightarrow x
$$

which is orthogonal. Since a most general sort assignment cannot exclude variable overlaps of the first rule with itself, Theorem 43 can only succeed when used in combination with an LL-labeling. Note that all preconditions for Corollaries 16, 25, and 31 are satisfied and due to the lack of critical overlaps they are decreasing. For the same reason Theorem 56 applies.

The final example shows that Theorem 56 does not subsume the plain version for linear TRSs (because of the variable condition).

Example 63 Consider the linear TRS consisting of the single rule

$$
(x+y)+z \rightarrow(z+y)+x
$$

Note that all steps are labeled the same, because they use the same rule. There is only one (parallel) critical peak, $((z+y)+x)+u \leftarrow((x+y)+z)+u \rightarrow(u+z)+(x+y)$, which may be joined as $((z+y)+x)+u \rightarrow((x+y)+z)+u \leftarrow(u+z)+(x+y)$. Confluence of $\mathcal{R}$ can be established by Theorem 6 using the rule labeling from Lemma 7. On the other hand, trying to use Theorem 56 fails for this joining sequence, because $\mathcal{V} \operatorname{ar}(((z+y)+x)+u) \nsubseteq$ $\mathcal{V} \operatorname{ar}((z+y)+x)$. All other ways of joining the critical peak fail to be decreasing because they require more than one parallel rewrite step from $((z+y)+x)+u$ or $(u+z)+(x+y)$, e.g. $((z+y)+x)+u \rightarrow((x+y)+z)+y \rightarrow(y+z)+(x+y)$. 


\subsection{Related work}

In this section we relate our results to $[1,11]$.

To compare our setting with the main result from [11] we define the critical pair steps $\operatorname{CPS}(\mathcal{R})=\{s \rightarrow t, s \rightarrow u \mid t \leftarrow s \rightarrow u$ is a critical peak of $\mathcal{R}\}$. Furthermore let $\operatorname{CPS}^{\prime}(\mathcal{R})$ be the critical pair steps which do not give rise to trivial critical pairs.

Theorem 64 ([11, Theorem 3]) A left-linear locally confluent TRS $\mathcal{R}$ is confluent if $\operatorname{CPS}^{\prime}(\mathcal{R}) / \mathcal{R}$ is terminating.

Using the weak LL-labeling $\ell_{\mathrm{rt}}^{\mathrm{PCPS}^{\prime}(\mathcal{R})}$, from Theorem 56 we obtain the following corollary. Here $\operatorname{PCPS}^{\prime}(\mathcal{R})$ are the parallel critical pair steps which do not give rise to trivial parallel critical pairs.

Corollary 65 A left-linear TRS $\mathcal{R}$ whose parallel critical pairs are joinable is confluent if $\operatorname{PCPS}^{\prime}(\mathcal{R}) / \mathcal{R}$ is terminating.

Proof We need to show that the relative termination assumption eliminates the variable condition in Theorem 56. If PCPS $(\mathcal{R}) / \mathcal{R}$ is terminating then for any (non-trivial) parallel critical peak $t{ }_{\Gamma}^{P}+t \rightarrow \rightarrow_{\Delta} u$ we obtain $t \rightarrow_{\vee \Gamma}^{*} \cdot{ }_{\vee}{ }_{\Delta}^{*} \leftarrow u$, hence $Q$ can be chosen to be empty and $\varnothing=\mathcal{V} \operatorname{ar}\left(\left.v\right|_{\varnothing} \subseteq \mathcal{V} \operatorname{ar}\left(\left.s\right|_{P}\right)\right.$ trivially holds. ${ }^{4}$

We stress that despite the fact that the preconditions in Corollary 65 require more (implementation) effort to check than those in Theorem 64, in theory Corollary 65 subsumes Theorem 64. To this end observe that termination of $\operatorname{PCPS}^{\prime}(\mathcal{R}) / \mathcal{R}$ is equivalent to termination of $\mathrm{CPS}^{\prime}(\mathcal{R}) / \mathcal{R}$. Furthermore joinability of the parallel critical pairs is a necessary condition for confluence just as local confluence is.

Due to the flexibility of the $\ell_{\mathrm{rt}}^{\mathcal{S}}$ labeling we can also choose $\mathcal{S}$ to be (a subset of) the critical diagram steps $\operatorname{CDS}(\mathcal{R})=\left\{s \rightarrow t_{i}, s \rightarrow u_{j} \mid t_{0} \leftarrow s \rightarrow u_{0}\right.$ is a critical peak in $\mathcal{R}, t_{0} \rightarrow^{*} t_{n}=u_{m}{ }^{*} \leftarrow u_{0}, 0 \leqslant i \leqslant n$, and $\left.0 \leqslant j \leqslant m\right\}$. Using $\operatorname{CDS}(\mathcal{R})$ allows to detect a possible decrease also somewhere in the joining part of the diagrams. ${ }^{5}$ This incorporates (and generalizes) the idea of critical valleys [18]. However, we remark that our setting does not (yet) follow another recent trend, i.e., to drop development closed critical pairs (see [10, 18]). We leave this for future work.

Next we show that Corollary 25 generalizes the results from [1, Sections 5 and 6]. It is not difficult to see that the encoding presented in [1, Theorem 5.4] can be mimicked by Corollary 25 where linear polynomial interpretations over $\mathbb{N}$ of the shape as in (1)

$$
\begin{array}{ll}
\text { (1) } f_{i \mathbb{N}}(x)=x+c_{f} & \text { (2) } f_{i \mathbb{N}}(x)=x+c_{f_{i}}
\end{array}
$$

are used to prove termination of $\star(\mathcal{R})$ and $\ell_{\star} \times \ell_{\mathrm{rl}}$ is employed to show LL-decreasingness of the critical peaks. In contrast to [1, Theorem 5.4], which explicitly encodes these constraints in a single formula of linear arithmetic, our abstract formulation has the following advantages. First, we do not restrict to weight functions but allow powerful machinery for

\footnotetext{
${ }^{4}$ The condition that $\{s \rightarrow t \mid u \leftarrow s \rightarrow t$ is a critical pair $\} / \mathcal{R}$ is terminating also eliminates the variable condition.

${ }^{5}$ In [31] we employed the strictly weaker system where all steps of the join (e.g., $\left.t_{i} \rightarrow t_{i+1}\right)$ are used whereas here we use $s \rightarrow t_{i+1}$.
} 
proving relative termination and second our approach allows to combine arbitrarily many labelings lexicographically (cf. Lemma 14). Furthermore we stress that our abstract treatment of $\star(\mathcal{R})$ allows to implement Corollary 25 based on $\star(\mathcal{R})$ (cf. Section 6) which admits further gains in power (cf. Example 1 as well as Section 7).

The idea of the extension presented in [1, Example 6.1] amounts to using $\ell_{\mathrm{rl}} \times \ell_{\star}$ instead of $\ell_{\star} \times \ell_{\mathrm{rl}}$, which is an application of Lemma 14 in our setting. Finally, the extension discussed in [1, Example 6.3] suggests to use linear polynomial interpretations over $\mathbb{N}$ of the shape as in (2) to prove termination of $\star(\mathcal{R})$. Note that these interpretations are still weight functions. This explains why the approach from [1] fails to establish confluence of the TRSs in Examples 18 and 20 since a weight function cannot show termination of the rules $\mathrm{f}_{1}\left(\mathrm{~g}_{1}(x)\right) \rightarrow \mathrm{g}_{1}\left(\mathrm{f}_{1}(x)\right)$ and $\mathrm{f}_{1}\left(\mathrm{~h}_{1}(x)\right) \rightarrow \mathrm{h}_{1}\left(\mathrm{~g}_{1}\left(\mathrm{f}_{1}(x)\right)\right)$, respectively.

Note that both recent approaches $[1,11]$ based on decreasing diagrams fail to prove the TRS $\mathcal{R}$ from Example 1 confluent. The former can, e.g., not cope with the nonterminating rule $\times_{1}(x) \rightarrow+_{0}\left(\times_{1}(x)\right)$ in $\mathcal{R}_{>}^{\star}$ (cf. Example 28) while overlaps with the non-terminating rule $x+y \rightarrow y+x \in \mathcal{R}$ prevent the latter approach from succeeding. In contrast, Examples 17 and 28 give two confluence proofs based on our setting.

\section{Implementation}

In this section we sketch how the results from this article can be implemented.

Before decreasingness of critical peaks can be investigated, the critical pairs must be shown to be convergent. For a critical pair $t \leftarrow \rtimes \rightarrow u$ in our implementation we consider all joining sequences such that $t \rightarrow \leqslant n . \leqslant n \leftarrow u$ and there is no smaller $n$ that admits a common reduct. While in theory longer joining sequences might be easier to label decreasingly, preliminary experiments revealed that the effort due to the consideration of additional diagrams decreased performance.

To exploit the possibility for incremental confluence proofs by lexicographically combining labels (cf. Lemmata 9 and 14) our implementation considers lists of labels. The search for relative termination proofs (and thus the labelings) is implemented by encoding the constraints in non-linear (integer) arithmetic. Below we describe how we combine existing labels (some partial progress) with the search for a new labeling to show the critical peaks decreasing. Note that labelings use different domains (natural numbers, terms), and, even worse, different orders (matrix interpretations, LPO, etc.). The crucial observation for incremental labeling is that neither the actual labels nor the precise order on the labels have to be recorded but only how the labels in the join relate to the labels from the peak. We use the following encoding. Let the local peak have labels $t_{\alpha} \leftarrow s \rightarrow_{\beta} u$. Then a step $v \rightarrow_{\gamma} w$ is labeled by the pair $\left(\circ_{\alpha}, o_{\beta}\right)$ where $\circ_{\alpha}$ and $o_{\beta}$ indicates if $\alpha \circ_{\alpha} \gamma$ and $\beta \circ_{\beta} \gamma$, respectively. Here $\left\{\circ_{\alpha}, o_{\beta}\right\} \subseteq\{>, \geqslant, ?\}$ and ? means that the labels are incomparable, e.g., $\mathrm{f}(x)$ ? $\mathrm{g}(y)$ in LPO or $2 x+1 ? x+2$ for (matrix) interpretations. ${ }^{6}$ Decreasingness as depicted in Fig. 8a can then be captured by the conditions shown in Fig. 8b, where $\circ$ can be replaced by any symbol.

It is straightforward to implement Corollary 16. After establishing termination of $\mathcal{R}_{d} / \mathcal{R}_{n d}$ (e.g., by an external termination prover) any weak LL-labeling can be tried to show the critical peaks decreasing. In $[1,11]$ it is shown how the rule labeling can be implemented by encoding the constraints in linear arithmetic. Note that when using weak LL-labelings

\footnotetext{
${ }^{6}$ Our previous implementation (reported in [31]) had a bug, as it did not track incomparable labels properly.
} 


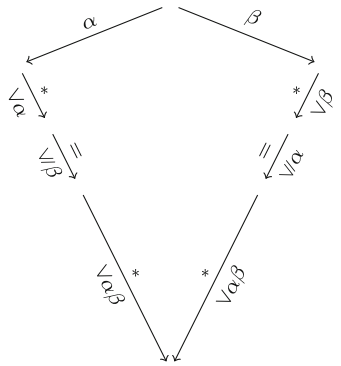

(a) Decreasingness

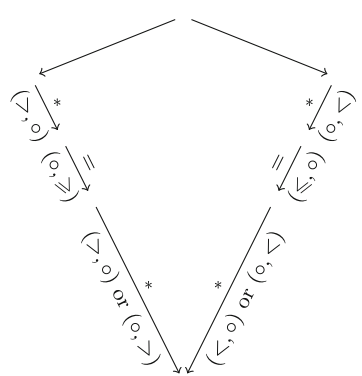

(b) Encoding of decreasingness

Fig. 8 Encoding the order on the labels

the implementation does not have to test condition 2 in Definition 10 since this property is intrinsic to weak LL-labelings.

We sketch how to implement the labeling $\ell_{\mathrm{rt}}^{\mathcal{S}}$ from Lemma 8 as a relative termination problem. First we fix a suitable set $\mathcal{S}$, i.e., the critical diagram steps (see Section 5). Facing the relative termination problem $\mathcal{S} / \mathcal{R}$ we try to simplify it according to Theorem 2 into some $\mathcal{S}^{\prime} / \mathcal{R}^{\prime}$. Note that it is not necessary to finish the proof. By Theorem 2 the relative TRS $\left(\mathcal{S} \backslash \mathcal{S}^{\prime}\right) / \mathcal{R}$ is terminating and hence by Lemma $8 \ell_{\mathrm{rt}}^{\mathcal{S} \backslash \mathcal{S}^{\prime}}$ is an L-labeling. Let $\geqslant=\rightarrow_{\mathcal{R}}^{*}$ and $>=\rightarrow_{\left(\mathcal{S} \backslash \mathcal{S}^{\prime}\right) / \mathcal{R}}^{+}$. Since $\geqslant$and $>$can never increase by rewriting, it suffices to exploit the first decrease with respect to $>$. Consider a rewrite sequence $v_{1} \rightarrow_{\mathcal{R}} v_{2} \rightarrow_{\mathcal{R}} \cdots \rightarrow_{\mathcal{R}} v_{l}$. Take the smallest $k$ such that $v_{1} \rightarrow v_{k+1} \in \mathcal{S}$ but $v_{1} \rightarrow v_{k+1} \notin \mathcal{S}^{\prime}$. Then $v_{i} \rightarrow(\geqslant, \geqslant) v_{i+1}$ for $1 \leqslant i \leqslant k$ and $v_{i} \rightarrow_{(>,>)} v_{i+1}$ for $k<i<l$. If no such $k$ exists set $v_{i} \rightarrow_{(\geqslant, \geqslant)} v_{i+1}$ for $1 \leqslant i<l$. We demonstrate the above idea on an example.

Example 66 Consider the following TRS $\mathcal{R}$ from [4]:

$$
\mathrm{I}(x) \rightarrow \mathrm{I}(\mathrm{J}(x)) \quad \mathrm{J}(x) \rightarrow \mathrm{J}(\mathrm{K}(\mathrm{J}(x))) \quad \mathrm{H}(\mathrm{I}(x)) \rightarrow \mathrm{K}(\mathrm{J}(x)) \quad \mathrm{J}(x) \rightarrow \mathrm{K}(\mathrm{J}(x))
$$

We show how the critical peak $\mathrm{H}(\mathrm{I}(\mathrm{J}(x))) \leftarrow \mathrm{H}(\mathrm{I}(x)) \rightarrow \mathrm{K}(\mathrm{J}(x))$ can be closed decreasingly $\mathrm{H}(\mathrm{I}(\mathrm{J}(x))) \rightarrow(\geqslant, \geqslant) \mathrm{K}(\mathrm{J}(\mathrm{J}(x))) \rightarrow{ }_{(>,>)} \mathrm{K}(\mathrm{J}(\mathrm{K}(\mathrm{J}(x))))_{(\leqslant, \leqslant)} \leftarrow \mathrm{K}(\mathrm{J}(x))$ by $\ell_{\mathrm{rt}}^{\mathcal{S}}$. Let $\mathcal{S}$ be the TRS consisting of the critical diagram steps from the above diagram, i.e.,

$$
\begin{array}{ll}
\mathrm{H}(\mathrm{I}(x)) \rightarrow \mathrm{H}(\mathrm{I}(\mathrm{J}(x))) & \mathrm{H}(\mathrm{I}(x)) \rightarrow \mathrm{K}(\mathrm{J}(\mathrm{J}(x))) \\
\mathrm{H}(\mathrm{I}(x)) \rightarrow \mathrm{K}(\mathrm{J}(x)) & \mathrm{H}(\mathrm{I}(x)) \rightarrow \mathrm{K}(\mathrm{J}(\mathrm{K}(\mathrm{J}(x))))
\end{array}
$$

The interpretation $\mathrm{H}_{\mathbb{N}}(x)=\mathrm{J}_{\mathbb{N}}(x)=\mathrm{K}_{\mathbb{N}}(x)=x$ and $\mathrm{I}_{\mathbb{N}}(x)=x+1$ allows to "simplify" termination of the problem $\mathcal{S} / \mathcal{R}$ according to Theorem 2 . Since the rules that reduce the number of I's are dropped from $\mathcal{S}$ (and $\mathcal{R}$ ), those rules admit a decrease in the labeling.

The abstraction works similarly for the labelings $\ell_{\star}$ and $\ell_{\triangle}$ from Lemmata 23 and 29, respectively.

Finally, we explain why $\star(\mathcal{R})$ need not be computed explicitly to implement Corollary 25 with the labeling from Lemma 27 . The idea is to start with $\star(\mathcal{R})$ and incrementally prove termination of $\mathcal{R}_{>}^{\star} / \mathcal{R}_{=}^{\star}$ until some $\mathcal{S}_{1} / \mathcal{S}_{2}$ is reached. If all left-hand sides in $\mathcal{S}_{1}$ are distinct then they must have been derived from different combinations $(l, x)$ with $l \rightarrow r \in \mathcal{R}$ and 
Table 1 Experimental results for 92 left-linear TRSs

\begin{tabular}{|c|c|c|c|c|}
\hline method & pre & $\mathrm{CR}\left(\ell_{\mathrm{rl}}\right)$ & $\mathrm{CR}\left(\ell_{\mathrm{rt}}\right)$ & $\mathrm{CR}$ \\
\hline Theorem 6 & 69 & 42 & 36 & 44 \\
\hline Theorem 41 & 92 & 46 & 40 & 48 \\
\hline Theorem 43 & 92 & 53 & - & - \\
\hline Corollary 16 & 65 & 47 & 40 & 49 \\
\hline Corollary $25 \star$ & 66 & 48 & 41 & 50 \\
\hline Corollary $25_{\star}^{\star}$ & 69 & 51 & 43 & 53 \\
\hline Corollary 31 & 65 & 47 & 41 & 49 \\
\hline Theorem 56 & 92 & 55 & 55 & 57 \\
\hline
\end{tabular}

$x \in \mathcal{V} \operatorname{ar}(l) .{ }^{7}$ Hence they are exactly those rules which should be placed in $\mathcal{R}_{=}^{\star}$. We show the idea by means of an example.

Example 67 We revisit Example 1 and try to prove termination of $\star(\mathcal{R})$. By an application of Theorem 2 with the interpretation given in Example 28 the problem is termination equivalent to $\mathcal{R}_{\dagger} / \mathcal{R}_{=}^{\star}$. By another application of Theorem 2 the same proof can be used to show termination of $\left(\mathcal{R}_{>}^{\star} \backslash \mathcal{R}_{\dagger}^{\star}\right) /\left(\mathcal{R}_{=}^{\star} \cup \mathcal{R}_{+}^{\star}\right)$ which is a suitable candidate for $\star(\mathcal{R})$ since the rules in $\mathcal{R}_{\dagger}^{\star}$ have different left-hand sides.

We have also implemented Theorems 41 and 43. The requirements of Theorem 41 can be checked effectively by the following characterization of $t \in \mathcal{T}_{\unlhd \alpha}(\mathcal{F}, \mathcal{V})$ :

Remark 68 The condition $t \in \mathcal{T}_{\unlhd \alpha}(\mathcal{F}, \mathcal{V})$ holds if and only if $t$ is $S$-sorted and $S(t)\left(\leq \cup \triangleleft_{1}\right)^{*} \alpha$, where the relation $\triangleleft_{1}$ on sorts relates argument types to result types: $S(f, i) \triangleleft_{1} S(f)$ for all function symbols $f \in \mathcal{F}$ of arity $n$ and $1 \leqslant i \leqslant n$.

We only implemented the simplest case of Theorem 43, where $\ell$ is a rule labeling. First, using Remark 68, we determine for which rules $l \rightarrow r \in \mathcal{R}, l^{\prime} \rightarrow r^{\prime} \in \mathcal{R}$, it is possible to nest $l^{\prime} \rightarrow r^{\prime}$ below a duplicating variable of $l \rightarrow r$. We add constraints $i(l \rightarrow r)>i\left(l^{\prime} \rightarrow r^{\prime}\right)$ to our constraint satisfaction problem for the rule labeling. The hard work is done by an SMT solver.

To postpone the expensive computation (and labeling) of parallel critical pairs as long as possible we implemented Theorem 56 according the following lazy approach. We first find ordinary weak LL-labelings for the critical diagrams, as described earlier in this section. Only if confluence cannot be established by considering this weak LL-labeling for (non-parallel) critical peaks, we generate parallel critical peaks together with joining sequences. Finally, we check whether the weak LL-labeling joins all resulting diagrams (critical and parallel critical) decreasingly as per Theorem 56. This check is also responsible for combining single steps into a parallel one for the joining sequence. We confess that

\footnotetext{
${ }^{7}$ When computing $\star(\mathcal{R})$ the implementation renames variables such that $(\ell, x)$ uniquely identifies a rule $\ell \rightarrow r$
} 
Table 2 Comparison with other tools on 92 left-linear TRSs

\begin{tabular}{lll}
\hline tool & CR & not CR \\
\hline ACP & 63 & 22 \\
CSI & 67 & 20 \\
saigawa & 53 & 12 \\
\hline$\sum$ & 68 & 22 \\
\hline
\end{tabular}

this implementation for Theorem 56 is somewhat opportunistic but allows to reuse partial progress (the weak LL-labeling) while postponing parallel critical pairs as long as possible.

\section{Experiments}

The results from the article have been implemented and form the core of the confluence prover CSI [30]. For experiments ${ }^{8}$ using version 0.4 of the tool we considered the current 276 TRSs in Cops. In the experiments we focus on the 149 systems which have been referenced from the confluence literature. From these systems 92 are left-linear. Our experiments have been performed on a notebook equipped with an Intel ${ }^{\circledR}$ quad core processor i7-2640M running at a clock rate of $2.8 \mathrm{GHz}$ and $4 \mathrm{~GB}$ of main memory.

For 3 systems not even local confluence could be established within a time limit of 60 seconds. All other tests finished within this time limit.

Table 1 shows an evaluation of the results from this article. The first column indicates which criterion has been used to investigate confluence. $A \star$ means that the corresponding corollary is implemented using $\star(\mathcal{R})$ whereas $\star$ refers to $\star(\mathcal{R})$. The column labeled pre shows for how many systems the precondition of the respective criterion is satisfied, e.g., for Theorem 6 the precondition is linearity while for Corollary 16 the precondition is termination of $\mathcal{R}_{d} / \mathcal{R}_{n d}$. The columns labeled $\mathrm{CR}(\ell)$ give the number of systems for which confluence could be established using labeling $\ell$. (For Corollary 25 implicitly $\ell_{\star}$ is also employed. Similarly Corollary 31 employs $\ell_{\triangle}$.) The column labeled CR corresponds to the full power of each result, i.e., when the lexicographic combination of all labelings is used.

From the table we draw the following conclusions. On this test bed the labeling function $\ell_{\mathrm{rl}}$ can handle more systems than $\ell_{\mathrm{rt}}$ when considering single steps but for parallel rewriting both labelings succeed on equally many systems. Still, in both settings most power is obtained when using all labelings. In practice the study of parallel rewriting (Theorem 56) is beneficial. This suggests that the preconditions to obtain weak LL-labelings are severe.

For reference in Table 2 we compare the power of the confluence provers participating in the Confluence Competition (CoCo), ${ }^{9}$ i.e., ACP [4], CSI [30], and saigawa [11, 13].

- ACP is a powerful confluence prover which implements numerous confluence criteria from the literature. Its distinctive feature is the strong support for problems with AC semantics [2].

\footnotetext{
${ }^{8}$ Details available from http://cl-informatik.uibk.ac.at/software/csi/labeling2.

${ }^{9}$ http://coco.nue.riec.tohoku.ac.jp
} 
- CSI gains most of its power from the labeling framework presented here. In addition it implements development closed critical pairs [19] and persistence [7]. Recently, the techniques introduced in [2] and [13] have also been integrated.

- saigawa also heavily exploits relative termination, remarkably also to analyze confluence of non-left-linear systems [13].

From Tables 1 and 2 we conclude that our framework admits a state-of-the-art confluence prover for left-linear systems.

\section{Conclusion}

In this article we studied how the decreasing diagrams technique can be automated. We presented conditions (subsuming recent related results) that ensure confluence of a left-linear TRS whenever its critical peaks are decreasing. The labelings we proposed can be combined lexicographically which allows incremental proofs of confluence and has a modular flavor in the following sense: Whenever a new labeling function is invented, the whole framework gains power. We discussed several situations (Examples 1, 18, 20,60) where traditional confluence techniques fail but our approach easily establishes confluence.

We have also considered parallel rewriting resulting in a significantly more powerful approach. We leave the study of $\rightarrow$ and the integration of development closed critical pairs as in $[10,18]$ as future work.

Recently confluence by decreasing diagrams (for abstract rewrite systems) has been formalized in the theorem prover Isabelle/HOL [28, 29]. Since the generated (incremental) labeling proofs are often impossible to check for humans it seems a natural point for future work to also formalize the labeling framework to enable automatic certification of confluence proofs. Since our setting is based on a single method (decreasing diagrams) while still powerful it offers itself as a perfect candidate for future certification efforts.

Acknowledgments We thank the anonymous reviewers for providing many helpful and detailed comments.

Open Access This article is distributed under the terms of the Creative Commons Attribution License which permits any use, distribution, and reproduction in any medium, provided the original author(s) and the source are credited.

\section{References}

1. Aoto, T.: Automated confluence proof by decreasing diagrams based on rule-labelling. In: Proceedings of the 21st International Conference on Rewriting Techniques and Applications. Leibniz International Proceedings in Informatics 6, 7-16 (2010)

2. Aoto, T., Toyama, Y.: A reduction-preserving completion for proving confluence of non-terminating term rewriting systems. Logical Methods in Computer Science 8(1:31), 1-29 (2012)

3. Aoto, T., Toyama, Y.: Persistency of confluence. Journal of Universal Computer Science 3(11), 1134 1147 (1997)

4. Aoto, T., Yoshida, J., Toyama, Y.: Proving confluence of term rewriting systems automatically. In: Proceedings of the 20th International Conference on Rewriting Techniques and Applications. Lect. Notes Comput. Sci. 5595, 93-102 (2009)

5. Baader, F., Nipkow, T.: Term Rewriting and All That. Cambridge University Press (1998)

6. Felgenhauer, B.: Rule labeling for confluence of left-linear term rewrite systems. In: Proceedings of the 2nd International Workshop on Confluence, pp. 23-27 (2013) 
7. Felgenhauer, B., Zankl, H., Middeldorp, A.: Proving confluence with layer systems. In: Proceedings of the 31st IARCS Annual Conference on Foundations of Software Technology and Theoretical Computer Science. Leibniz International Proceedings in Informatics 13, 288-299 (2011)

8. Geser, A. Relative termination. Ph.D. thesis, Universität Passau (1990). Available as technical report 91-03

9. Gramlich, B.: Confluence without termination via parallel critical pairs. In: Proceedings of the 21st International Colloquium on Trees in Algebra and Programming. Lect. Notes Comput. Sci. 1059, 211225 (1996)

10. Hirokawa, N., Middeldorp, A.: Commutation via relative termination. In: Proceedings of the 2nd International Workshop on Confluence, pp. 29-33 (2013)

11. Hirokawa, N., Middeldorp, A.: Decreasing diagrams and relative termination. J. Autom. Reason. 47(4), 481-501 (2011)

12. Huet, G.: Confluent reductions: Abstract properties and applications to term rewriting systems. J. ACM 27(4), 797-821 (1980)

13. Klein, D., Hirokawa, N.: Confluence of non-left-linear TRSs via relative termination. In: Proceedings of the 18th International Conference on Logic for Programming, Artificial Intelligence, and Reasoning. Lect. Notes Comput. Sci. 7180, 258-273 (2012). (Advanced Research in Computing and Software Science)

14. Knuth, D., Bendix, P.: Simple word problems in universal algebras. In: Leech, J. (ed.) Computational Problems in Abstract Algebra, pp. 263-297. Pergamon Press (1970)

15. Okui, S.: Simultaneous critical pairs and Church-Rosser property. In: Proceedings of the 9th International Conference on Techniques, Rewriting, Applications. Lect. Notes Comput. Sci. 1379, 2-16 (1998)

16. van Oostrom, V.: Confluence by decreasing diagrams. Theor. Comput. Sci. 126(2), 259-280 (1994)

17. van Oostrom, V.: Confluence by decreasing diagrams - converted. In: Proceedings of the 19th International Conference on Rewriting Techniques and Applications. Lect. Notes Comput. Sci. 5117, 306-320 (2008)

18. van Oostrom, V.: Confluence via critical valleys. In: Proceedings 6th International Workshop on HigherOrder Rewriting, pp. 9-11 (2012)

19. van Oostrom, V.: Developing developments. Theor. Comput. Sci. 175(1), 159-181 (1997)

20. Oyamaguchi, M., Ohta, Y.: A new parallel closed condition for Church-Rosser of left-linear term rewriting systems. In: Proceedings of the 8th International Conference on Rewriting Techniques and Applications. Lect. Notes Comput. Sci. 1232(1997), 187-201

21. Rosen, B.: Tree-manipulating systems and Church-Rosser theorems. J. ACM 20(1), 160-187 (1973)

22. Stump, A., Zantema, H., Kimmell, G., Omar, R.: A rewriting view of simple typing. Logical Methods in Computer Science 9(1:4), 1-29 (2012)

23. Terese: Term Rewriting Systems, Cambridge Tracts in Theoretical Computer Science, vol. 55. Cambridge University Press (2003)

24. Toyama, Y.: Commutativity of term rewriting systems. In: Fuchi, K., Kott, L. (eds.) Programming of Future Generation Computers II, pp. 393-407. North-Holland (1988)

25. Toyama, Y.: On the Church-Rosser property for the direct sum of term rewriting systems. J. ACM 34(1), 128-143 (1987)

26. Toyama, Y.: On the Church-Rosser property of term rewriting systems. Tech. Rep. 17672 (1981). NTT ECL

27. Waldmann, U.: Semantics of order-sorted specifications. Theor. Comput. Sci. 94(1), 1-35 (1992)

28. Zankl, H.: Confluence by decreasing diagrams - formalized. In: Proceedings of the 24th International Conference on Rewriting Techniques and Applications. Leibniz International Proceedings in Informatics 21, 352-367 (2013)

29. Zankl, H. Decreasing diagrams. Archive of Formal Proofs (2013). Formal proof development, http://afp. sf.net/entries/Decreasing-Diagrams.shtml

30. Zankl, H., Felgenhauer, B., Middeldorp, A.: CSI - A confluence tool. In: Proceedings of the 23rd International Conference on Deduction, Automated. Lecture Notes in Artificial Intelligence 6803, 499-505 (2011)

31. Zankl, H., Felgenhauer, B., Middeldorp, A.: Labelings for decreasing diagrams. In: Proceedings of the 22nd International Conference on Rewriting Techniques and Applications. Leibniz International Proceedings in Informatics 10, 377-392 (2011) 